\title{
Effect of Raw Material, Pressing and Glycosidase on the Volatile Compound Composition of Wine Made From Goji Berries
}

\author{
Guanshen Yuan ${ }^{1}$, Jie Ren ${ }^{1}$, Xiaoyu Ouyang ${ }^{1}$, Liying Wang ${ }^{2}$, Mengze Wang ${ }^{2}$, Xiaodong Shen ${ }^{2}$, \\ Bolin Zhang ${ }^{1}$ and Baoqing Zhu ${ }^{1, *}$ \\ 1 Beijing Key Laboratory of Forestry Food Processing and Safety, Department of Food Science, \\ College of Biological Sciences and Biotechnology, Beijing Forestry University, Beijing 100083, China; \\ guanshenyuan@126.com (G.Y.); chxdyd2012@163.com (J.R); oyxy1993@sina.com (X.O.); \\ zhangbolin888@163.com (B.Z) \\ 2 Ningxia Senmiao Goji Technology Development Co. Ltd., Yinchuan, Ningxia 750000, China; \\ wangliying@senmiao.com (L.W.); wangmengze@senmiao.com (M.W.); shenxiaodong@senmiao.com (X.S.) \\ * Correspondence: zhubaoqing@bjfu.edu.cn; Tel.: +86-10-62338221
}

Academic Editor: Derek J. McPhee

Received: 2 August 2016; Accepted: 28 September 2016; Published: 2 October 2016

\begin{abstract}
This study investigated the effect of raw material, pressing, and glycosidase on the aromatic profile of goji berry wine. The free-run and the pressed juice of dried and fresh goji berries were used for wine production, whereas glycosidase was applied to wine after fermentation. Dried goji berry fermented wine exhibited much stronger fruity, floral, caramel, and herbaceous odors due to higher levels of esters, $\beta$-ionone and methionol. However, fresh berry fermented wine possessed stronger chemical notes due to higher levels of 4-ethylphenol. Pressing treatment reduced the fruity and caramel odors in these fermented wines, and fresh berry free-run juice fermented wine exhibited the least floral aroma. Glycosidase addition did not alter the aromatic composition of wines. The principal component analysis indicated that goji raw material played a primary role in differentiating the aromatic profiles of the wines due to the difference on the content of 20 esters, nine benzenes, eight aldehydes/ketones, three acids, two alcohols and six other volatiles. The content differences on isopentyl alcohol, styrene, benzyl alcohol, 1-octanol, (E)-5-decen-1-ol, 1-hexanol, and $\beta$-cyclocitral resulted in the segregation of the wines with and without the pressing treatment, especially for fresh berry fermented wine.
\end{abstract}

Keywords: goji wine; dried goji berry; fresh goji berry; pressing; free and pressed juice; glycosidase; aromatic compounds

\section{Introduction}

Goji (Lycium barbarum L.) is an important economic crop widely cultivated in the northwestern regions of China [1]. Goji berries are consumed as a functional food due to their multiple health properties [2]. They have been reported to have the capacity to decrease the incidence of vision, liver, kidney, and immune system problems because of their high levels of functional ingredients, such as polysaccharides, flavonoids and carotenoids [3-5].

Goji berries are indefinite inflorescences, and the fruits normally mature between June and August. Therefore, ripe goji berry fruits have to be harvested several times a year. Fresh goji berries readily spoil during storage, and therefore the majority (about 90\%) of fresh goji berries are normally processed into dried products. Additionally, the goji berry wine fermentation industry has attracted attention in China, since goji berry wine can preserve the nutritional value of goji bery and extend the shelf-life [6]. 
The overall aroma determines the sensory feature and quality of any fruit wine. The overall aroma in fruit wine is comprised of the primary, secondary, and tertiary aromas [7]. The primary aroma consists of volatile compounds derived from the fruit [7]. Fermentation results in the production of volatile compounds with fermented notes [8]. The evolution of volatiles during the aging process can bring aging odors to wine [8]. Fruit-derived aromatic compounds essentially determine the typical flavor of fruit wine [9]. Several strategies have been applied in the wine industry to investigate their effect on the extraction of fruit-derived volatiles. For example, pressing treatment is a physical approach to disintegrate the structure of fruit cells, enhancing the release of aromatic compounds and volatile precursors in juice. It has been reported that the pressed juice contained higher levels of $\mathrm{C}_{6}$ alcohols compared to the free-run juice [10]. Besides, odorless volatile precursors exist in fruit with a large amount [9]. Glycosidase can cleave the sugar moieties from bound volatiles to release free volatiles that can contribute to the overall aroma of wine [9,11-13]. For example, terpene alcohols are the major volatile aglycones released from their precursors by glycosidase in grape wine, and these aromatic compounds can improve the floral and fruity notes of the overall aroma of grape wine [9,11-13].

The effect of pressing and glycosidase on the evolution of volatile composition during grape wine production has been well studied $[9,14]$. However, such investigations on goji berry wine production have barely been documented. More importantly, fresh goji berry is always processed into a dried form. The drying process can cause dramatic changes in the volatile composition and aromatic precursors in dried goji berry, which may alter the aromatic profile in goji berry wine. Therefore, we selected dried and fresh goji berries, and applied pressing and glycosidase treatments during the goji berry wine-making process. The objective of this study was to investigate the effect of goji berry raw material, pressing treatment, and glycosidase application on the volatile composition of goji berry wine, which could provide practical knowledge on quality development of goji berry wine fermentation. More importantly, the findings from this study could also provide a useful reference on material selection and fermentation processing development using fruits different from grapes.

\section{Results and Discussion}

\subsection{Physicochemical Parameters of Goji Wine}

Alcohol strength, volatile acidity, titratable acidity, residual sugar, polysaccharide, free $\mathrm{SO}_{2}$, total $\mathrm{SO}_{2}$, and $\mathrm{pH}$ of these goji berry fruit wines are listed in Table 1 . These goji berry fruit wines contained the high level of the titratable acidity and the $\mathrm{pH}$ value, indicating that these wines exhibited a stronger buffer capacity. This wine feature might be attributed to that goji berry is cultivated in a high saline level soil condition.

Additionally, polysaccharide is an important bioactive component that is largely present in goji berry [15]. The level of polysaccharide in these goji berry wines ranged of 1-3 g/L, which was much higher than that in grape wines $(0.2-1.5 \mathrm{~g} / \mathrm{L})$ [16]. Meanwhile, the dried goji berry wine made from pressed juice showed the polysaccharide content about 2 times higher than the other goji berry wines, whereas the fresh goji berry pressed juice wine did not show any increase on the level of polysaccharide. These indicated that the drying process of goji berry fruit might cause the structure change of goji berry fruit or polysaccharide in fruit, which resulted in such differences on the polysaccharide level in the wines. 
Table 1. Physicochemical parameters of goji berry fermented wines.

\begin{tabular}{|c|c|c|c|c|c|c|c|c|}
\hline Oenological Parameters & DF & DF-G & DP & DP-G & FF & FF-G & FP & FP-G \\
\hline Alcohol $(\%, v / v)$ & $13.0 \pm 0.0 \mathrm{a}$ & $13.00 \pm 0.0 \mathrm{a}$ & $12.5 \pm 0.2 \mathrm{bcd}$ & $12.6 \pm 0.0 \mathrm{bc}$ & $12.4 \pm 0.1 \mathrm{~cd}$ & $12.7 \pm 0.0 \mathrm{~b}$ & $12.4 \pm 0.0 \mathrm{~d}$ & $12.3 \pm 0.0 \mathrm{~d}$ \\
\hline Volatile acidity (g/L) & $1.2 \pm 0.0 \mathrm{~b}$ & $1.2 \pm 0.0 \mathrm{bc}$ & $1.1 \pm 0.0 \mathrm{e}$ & $0.8 \pm 0.0 \mathrm{~g}$ & $1.2 \pm 0.0 \mathrm{~cd}$ & $1.0 \pm 0.0 \mathrm{f}$ & $1.1 \pm 0.0 \mathrm{de}$ & $2.1 \pm 0.0 \mathrm{a}$ \\
\hline 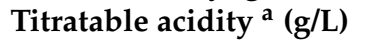 & $12.2 \pm 0.1 \mathrm{ab}$ & $11.9 \pm 0.1 \mathrm{bc}$ & $11.8 \pm 0.0 \mathrm{c}$ & $12.4 \pm 0.1 \mathrm{a}$ & $7.9 \pm 0.0 \mathrm{e}$ & $7.7 \pm 0.0 \mathrm{e}$ & $8.2 \pm 0.1 \mathrm{e}$ & $7.8 \pm 0.0 \mathrm{~d}$ \\
\hline Residual sugar (g/L) & $9.3 \pm 0.4 \mathrm{a}$ & $10.0 \pm 0.4 \mathrm{a}$ & $10.1 \pm 0.7 \mathrm{a}$ & $9.1 \pm 0.0 \mathrm{a}$ & $7.8 \pm 0.1 \mathrm{~b}$ & $6.6 \pm 0.0 \mathrm{~b}$ & $8.4 \pm 0.1 \mathrm{~b}$ & $7.6 \pm 0.1 b$ \\
\hline Polysaccharide $^{b}(g / L)$ & $1.6 \pm 0.1 \mathrm{~b}$ & $1.5 \pm 0.0 \mathrm{bc}$ & $3.0 \pm 0.1 \mathrm{a}$ & $2.9 \pm 0.1 \mathrm{a}$ & $1.4 \pm 0.0 \mathrm{~cd}$ & $1.2 \pm 0.0 \mathrm{df}$ & $1.3 \pm 0.1 \mathrm{~d}$ & $1.1 \pm 0.1 \mathrm{f}$ \\
\hline Free $\mathrm{SO}_{2}(\mathrm{mg} / \mathrm{L})$ & $3.4 \pm 0.0 \mathrm{c}$ & $1.7 \pm 0.1 \mathrm{~d}$ & $3.4 \pm 0.0 \mathrm{c}$ & $1.7 \pm 0.0 \mathrm{~d}$ & $13.4 \pm 0.0 \mathrm{a}$ & $7.3 \pm 0.4 b$ & $13.4 \pm 0.0 \mathrm{a}$ & $6.7 \pm 0.0 \mathrm{~b}$ \\
\hline Total $\mathrm{SO}_{2}(\mathrm{mg} / \mathrm{L})$ & $100.4 \pm 0.1 \mathrm{f}$ & $98.8 \pm 0.2 \mathrm{~g}$ & $93.7 \pm 0.0 \mathrm{~h}$ & $132.1 \pm 0.0 \mathrm{~d}$ & $210.5 \pm 0.2 b$ & $153.9 \pm 0.1 \mathrm{c}$ & $237.5 \pm 0.1 \mathrm{a}$ & $112.0 \pm 0.0 \mathrm{e}$ \\
\hline $\mathrm{pH}$ & $4.0 \pm 0.0 \mathrm{c}$ & $3.9 \pm 0.0 \mathrm{c}$ & $4.0 \pm 0.0 \mathrm{~b}$ & $4.0 \pm 0.0 \mathrm{~b}$ & $4.4 \pm 0.0 \mathrm{a}$ & $4.5 \pm 0.0 \mathrm{a}$ & $4.4 \pm 0.0 \mathrm{a}$ & $4.5 \pm 0.0 \mathrm{a}$ \\
\hline
\end{tabular}

DF and DP indicate dried goji berry fermented wine made of free-run and pressed juice, respectively. DF-G and DP-G represent dried goji berry fermented wine made of free-run and pressed juice after the glycosidase treatment, respectively. FF and FP are fresh goji berry fermented wine made of free-run and pressed juice, respectively. FF-G and FP-G stand for fresh goji berry fermented wine made of free-run and pressed juice after the glycosidase treatment, respectively. ${ }^{a}$ Expressed as g/L of malic acid. ${ }^{b}$ Expressed as g/L of glucose. Different letters in the same row means significant differences at $p \leq 0.05$. Data were expressed as the mean \pm standard deviation. 
The total $\mathrm{SO}_{2}$ content in these goji berry wines was much higher than that in goji berry fruits before the fermentation (Table 1). Potassium metabisulfite was added to terminate the fermentation process in this study, which resulted in the elevation of $\mathrm{SO}_{2}$ content in these wines. Meanwhile, the yeasts used in this study also possessed the ability of produce $\mathrm{SO}_{2}$ during the fermentation.

\subsection{Volatile Composition in Wine Made of Dried and Fresh Goji Berry}

The drying process has been reported to destroy the berry cell structure, resulting in the release of nutrients and enzymes [18-21]. These compounds further experience dehydrogenation, oxidation, degradation, and/or Maillard reactions, leading to the formation of a large number of volatiles [22]. It has been reported that the concentration of $\mathrm{C}_{6}$ alcohols and aldehydes, inducing the cut grass odor, significantly decreased in fruits during the drying process [23]. Meanwhile, some volatiles with spicy and ripe fruity notes were reported to be generated during the drying period, enhancing the odor complexity of dried fruit [23].

The volatile compounds identified in this work are listed in Table 2. A total of 97 volatile compounds were detected in the dried goji berry wine, whereas the fresh goji berry wine contained 94 volatile compounds (Table 3). Particularly, 2-acetylpyrrole, 3-phenyl-4-hydroxyacetophenone, isobutyl hexanoate, and cedrol were detected only in the dried goji berry wine, whereas E-5-decen-1-ol existed only in the fresh wine. In these wines, higher alcohols appeared to be the predominant volatiles in the goji berry wine, followed by esters, benzenes, and acids. However, aldehydes, ketones, terpenes, and norisoprenoids existed in these wines in low amounts.

In this study, the fresh and the dried goji berry fermented wines showed similar volatile compositions. However, the concentration of these volatiles in the wine samples exhibited significant differences (Tables 3 and 4). The fresh berry fermented wine showed higher total contents of aromatic compounds. A total of 56 volatiles displayed higher concentrations in the dried berry fermented wine, whereas the fresh goji berry fermented wine contained 31 volatiles that exhibited higher concentrations.

These goji berry wines also showed high level of volatile acidity. It has been reported that yeasts under high osmotic stress condition generated more acetic acid in ice wine during fermentation [17]. We speculated that osmotic stress induced by high level polysaccharide in goji berry fruits might stimulate the yeasts to yield more volatile acids in the wine. However, a further investigation should be conducted. 
Table 2. Volatile compounds identification and their linearity range.

\begin{tabular}{|c|c|c|c|c|c|c|c|c|}
\hline Volatile & $\mathrm{CN}^{\mathrm{a}}$ & RI & ID $^{b}$ & $\mathrm{QI}^{\mathrm{c}}$ & Standard & Quantitative Curve & $\mathbf{R}^{2}$ & Linearity Range $(\mu \mathrm{g} / \mathrm{L})$ \\
\hline Volatile acids & A & & & & & & & \\
\hline DMECA $^{d}$ & A1 & 1367.6 & B & 95 & 4-Ethylphenol & $y=233.46 x+1.5085$ & $\mathrm{R}^{2}=0.9967$ & $0.78-50.00$ \\
\hline Acetic acid & A2 & 1453.8 & $\mathrm{~A}$ & 43 & Acetic acid & $y=53.61 x-29.07$ & $\mathrm{R}^{2}=0.9948$ & $32.78-1049.00$ \\
\hline Butanoic acid & A3 & 1630.4 & $\mathrm{~A}$ & 60 & Butanoic acid & $\mathrm{y}=21574 \mathrm{x}+7.5319$ & $\mathrm{R}^{2}=0.9999$ & $126.88-1015.00$ \\
\hline$\alpha$-Methylbutyric acid & $\mathrm{A} 4$ & 1671.7 & B & 74 & Butanoic acid & $y=21814 x-0.9852$ & $\mathrm{R}^{2}=0.9994$ & $63.44-1015.00$ \\
\hline Octanoic acid & A5 & 2062.1 & $\mathrm{~A}$ & 60 & Octanoic acid & $y=7754.7 x+1401.7$ & $\mathrm{R}^{2}=0.9982$ & $2365.00-9460.00$ \\
\hline Decanoic acid & A6 & 2271.8 & A & 60 & Decanoic acid & $y=55699 x-252.24$ & $\mathrm{R}^{2}=0.9609$ & $1147.50-9180.00$ \\
\hline Higher alcohols & $\mathrm{C}$ & & & & & & & \\
\hline 2-Methyl-1-propanol & $\mathrm{C} 1$ & 1104.0 & A & 43 & 2-Methyl-1-propanol & $y=17537 x+5926.7$ & $\mathrm{R}^{2}=0.9861$ & $10250.00-82000.00$ \\
\hline 1-Butanol & $\mathrm{C} 2$ & 1158.1 & A & 56 & 1-Butanol & $y=17266 x+36.061$ & $\mathrm{R}^{2}=1$ & $299.69-1198.75$ \\
\hline Isopentyl alcohol & $\mathrm{C} 3$ & 1216.0 & A & 55 & Isopentyl alcohol & $y=9481.2 x-6073.4$ & $\mathrm{R}^{2}=0.9985$ & $35300.00-282400.00$ \\
\hline Pentyl alcohol & $\mathrm{C} 4$ & 1255.5 & $\mathrm{~B}$ & 42 & 1-Hexanol & $y=1162.6 x-1.2723$ & $\mathrm{R}^{2}=0.9999$ & $4.93-78.95$ \\
\hline 1-Hexanol & C5 & 1352.5 & $\mathrm{~A}$ & 56 & 1-Hexanol & $y=1166.2 x-2.2718$ & $\mathrm{R}^{2}=0.9999$ & $39.47-315.78$ \\
\hline (Z)-3-Hexen-1-ol & $\mathrm{C} 6$ & 1384.7 & A & 41 & (Z)-3-Hexen-1-ol & $y=4651.6 x-7.5812$ & $\mathrm{R}^{2}=0.9968$ & $32.62-260.94$ \\
\hline 2-Heptanol & $\mathrm{C} 7$ & 1320.0 & $\mathrm{~A}$ & 45 & 2-Heptanol & $y=210.83 x+0.0189$ & $\mathrm{R}^{2}=0.9944$ & $2.44-9.75$ \\
\hline 3-Ethoxy-1-propanol & $\mathrm{C} 8$ & 1376.9 & B & 31 & 1-Hexanol & $y=1161.1 x-1.192$ & $\mathrm{R}^{2}=0.9998$ & $9.87-78.95$ \\
\hline 1-Octen-3-ol & C9 & 1448.6 & A & 57 & 1-Octen-3-ol & $y=118.3 x-0.4557$ & $\mathrm{R}^{2}=0.9993$ & $3.22-25.78$ \\
\hline Sulcatol & $\mathrm{C} 10$ & 1461.0 & $\mathrm{~A}$ & 95 & Sulcatol & $y=514.36 x-60.573$ & $\mathrm{R}^{2}=0.9936$ & $0.69-11.00$ \\
\hline 2-Ethyl-1-hexanol & $\mathrm{C} 11$ & 1488.8 & $\mathrm{~A}$ & 27 & 2-Ethyl-1-hexanol & $y=89.473 x-0.447$ & $\mathrm{R}^{2}=0.9988$ & $0.44-14.06$ \\
\hline$\left[R-\left(R^{*}, R^{*}\right)\right]-2,3$-Butanediol & $\mathrm{C} 12$ & 1538.9 & $\mathrm{~B}$ & 45 & {$\left[S-\left(R^{*}, R^{*}\right)\right]-2,3-$ Butanediol } & $y=82896 x-16783$ & $\mathrm{R}^{2}=0.9993$ & $39781.25-636500.00$ \\
\hline 1-Octanol & $\mathrm{C} 13$ & 1557.0 & A & 56 & 1-Octanol & $y=182.89 x+0.2501$ & $\mathrm{R}^{2}=0.9996$ & $6.45-51.56$ \\
\hline$\left[S-\left(R^{*}, R^{*}\right)\right]-2,3$-Butanediol & $\mathrm{C} 14$ & 1574.9 & A & 45 & {$\left[S-\left(R^{*}, R^{*}\right)\right]-2,3-$ Butanediol } & $y=82896 x-16783$ & $\mathrm{R}^{2}=0.9993$ & $39781.25-636500.00$ \\
\hline trans-2-Octenol & $\mathrm{C} 15$ & 1616.0 & A & 57 & trans-2-Octenol & $y=258.79 x+0.2617$ & $\mathrm{R}^{2}=0.9977$ & $2.61-20.88$ \\
\hline 1-Nonanol & $\mathrm{C} 16$ & 1659.1 & B & 56 & Acetic acid & $y=10.169 x-0.0206$ & $\mathrm{R}^{2}=0.9856$ & $0.13-0.51$ \\
\hline Methionol & $\mathrm{C} 17$ & 1720.6 & A & 106 & Methionol & $y=34890 x-113.09$ & $\mathrm{R}^{2}=0.9962$ & $280.00-1120.00$ \\
\hline E-5-Decen-1-ol & $\mathrm{C} 18$ & 1795.2 & $\mathrm{~B}$ & 67 & 1-Dodecanol & $y=300.63 x+0.7633$ & $\mathrm{R}^{2}=0.9962$ & $2.52-20.16$ \\
\hline Phenylethyl alcohol & C19 & 1920.2 & A & 91 & Phenylethyl alcohol & $y=1833.3 x-518.58$ & $\mathrm{R}^{2}=0.9940$ & $2537.50-40600.00$ \\
\hline
\end{tabular}


Table 2. Cont

\begin{tabular}{|c|c|c|c|c|c|c|c|c|}
\hline Volatile & $\mathrm{CN}^{\mathrm{a}}$ & RI & ID $^{b}$ & $Q^{c}$ & Standard & Quantitative Curve & $\mathbf{R}^{2}$ & Linearity Range $(\mu \mathrm{g} / \mathrm{L})$ \\
\hline Aldehydes/Ketones & AK & & & & & & & \\
\hline \multicolumn{9}{|l|}{ Aldehydes } \\
\hline$\overline{\text { Nonanal }}$ & AK1 & 1399.2 & A & 57 & Nonanal & $y=97.464 x-2.3267$ & $\mathrm{R}^{2}=0.9929$ & $0.44-28.44$ \\
\hline Furfural & $\mathrm{AK} 2$ & 1471.8 & A & 96 & Furfural & $y=1798.6 x-2.8178$ & $\mathrm{R}^{2}=0.9987$ & $5.25-84.00$ \\
\hline Decanal & AK3 & 1504.4 & $\mathrm{~A}$ & 43 & Decanal & $y=120.85 x+0.8893$ & $\mathrm{R}^{2}=0.9878$ & $0.31-39.53$ \\
\hline Safranal & $\mathrm{AK} 4$ & 1656.8 & $\mathrm{~B}$ & 107 & $\alpha$-Terpineol & $y=34.957 x+0.2522$ & $\mathrm{R}^{2}=0.9950$ & $0.42-3.38$ \\
\hline & \multicolumn{7}{|c|}{ Ketones } & $4.98-39.84$ \\
\hline Isobutyl ketone & AK6 & 1194.0 & $\mathrm{~B}$ & 57 & $6 \mathrm{M} 5 \mathrm{H} 2 \mathrm{NE}$ & $y=243.64 x-5.3362$ & $\mathrm{R}^{2}=0.9960$ & $0.04-92.00$ \\
\hline $\mathrm{TMCHN}^{\mathrm{e}}$ & AK7 & 1325.0 & $\mathrm{~B}$ & 82 & $6 \mathrm{M} 5 \mathrm{H} 3 \mathrm{NE}$ & $y=275.14 x-6.922$ & $\mathrm{R}^{2}=0.9947$ & $0.72-11.50$ \\
\hline $6 \mathrm{M} 5 \mathrm{H} 2 \mathrm{NE}^{\mathrm{f}}$ & AK8 & 1344.7 & A & 43 & $6 \mathrm{M} 5 \mathrm{H} 4 \mathrm{NE}$ & $y=278.06 x-7.1512$ & $\mathrm{R}^{2}=0.9971$ & $0.36-23.00$ \\
\hline 2-Undecanone & AK9 & 1603.7 & $\mathrm{~B}$ & 58 & Dihydropseudoionone & $y=53.532 x+0.0331$ & $\mathrm{R}^{2}=0.9995$ & $0.93-7.44$ \\
\hline$\beta$-Cyclocitral & AK10 & 1630.9 & B & 137 & $\alpha$-Terpineol & $y=179.5 x+0.7247$ & $\mathrm{R}^{2}=0.9993$ & $1.91-7.63$ \\
\hline Dihydropseudoionone & AK11 & 1861.4 & $\mathrm{~A}$ & 43 & Dihydropseudoionone & $y=50.565 x+0.0693$ & $\mathrm{R}^{2}=0.9981$ & $0.23-14.88$ \\
\hline 2-Acetylpyrrole & AK12 & 1981.6 & $\mathrm{~B}$ & 94 & Hexanal & $y=1093.5 x-17.772$ & $\mathrm{R}^{2}=0.9994$ & $30.74-245.94$ \\
\hline TMHAAL $\mathrm{g}$ & AK13 & 2372.3 & B & 111 & 4-Ethylphenol & $y=312.57 x+0.2238$ & $\mathrm{R}^{2}=0.9959$ & $0.10-12.50$ \\
\hline Benzenes/Phenols & B & & & & & & & \\
\hline \multicolumn{9}{|l|}{ Benzenes } \\
\hline$\overline{\text { Isodurene }}$ & B1 & 1498.2 & $\mathrm{~B}$ & 119 & $p$-Cymene & $y=54.679 x-0.1459$ & $\mathrm{R}^{2}=0.9956$ & $0.41-3.28$ \\
\hline TEMLNE ${ }^{\mathrm{h}}$ & B2 & 1677.5 & B & 159 & Ethyl salicylate & $y=79.909 x+0.0671$ & $\mathrm{R}^{2}=0.9932$ & $0.53-17.00$ \\
\hline DPPXL ${ }^{i}$ & B3 & 1694.1 & B & 175 & Ethyl salicylate & $y=56.72 x+3.6823$ & $\mathrm{R}^{2}=0.9945$ & $8.50-34.00$ \\
\hline Naphthalene & B4 & 1756.0 & $\mathrm{~A}$ & 128 & Naphthalene & $y=10.996 x+0.5718$ & $\mathrm{R}^{2}=0.9915$ & $0.95-7.56$ \\
\hline TMDHPE ${ }^{j}$ & B5 & 1758.3 & $\mathrm{~B}$ & 157 & Ethyl salicylate & $y=88.273 x-0.0789$ & $\mathrm{R}^{2}=0.9900$ & $4.98-39.84$ \\
\hline $\mathrm{M}^{2} \mathrm{~PB}^{\mathrm{k}}$ & B6 & 1790.2 & B & 120 & Ethyl phenacetate & $y=35.188 x+0.2362$ & $\mathrm{R}^{2}=0.9924$ & $0.84-3.38$ \\
\hline Phenethyl acetate & B7 & 1826.3 & A & 104 & Phenethyl acetate & $y=69.63 x+5.0169$ & $\mathrm{R}^{2}=0.9996$ & $17.81-285.00$ \\
\hline$\beta$-Methylnaphthalene & B8 & 1870.0 & $\mathrm{~B}$ & 142 & Ethyl salicylate & $y=60.837 x+1.9511$ & $\mathrm{R}^{2}=0.9906$ & $2.13-34.00$ \\
\hline Benzyl alcohol & B9 & 1883.4 & $\mathrm{~A}$ & 79 & Benzyl alcohol & $y=3642.2 x+19.022$ & $\mathrm{R}^{2}=0.9979$ & $91.56-732.50$ \\
\hline Ethyl dihydrocinnamate & B10 & 1895.6 & B & 104 & Ethyl phenacetate & $y=29.931 x+0.4139$ & $\mathrm{R}^{2}=0.9944$ & $0.42-6.75$ \\
\hline$\alpha$-Methylnaphthalene & B11 & 1906.9 & B & 142 & Ethyl salicylate & $y=79.909 x+0.0671$ & $\mathrm{R}^{2}=0.9932$ & $0.53-17.00$ \\
\hline 4-Tolylcarbinol & B12 & 1981.6 & $\mathrm{~B}$ & 107 & 4-Ethylphenol & $y=391.78 x-0.0605$ & $\mathrm{R}^{2}=0.9863$ & $0.20-3.13$ \\
\hline Ethyl cinnamate & B13 & 2145.3 & $\mathrm{~B}$ & 131 & Ethyl phenacetate & $y=29.468 x+0.4874$ & $\mathrm{R}^{2}=0.9940$ & $0.84-6.75$ \\
\hline $3 \mathrm{P} 4 \mathrm{HP}^{1}$ & B14 & 2249.8 & $\mathrm{~B}$ & 197 & Ethyl phenacetate & $y=34.957 x+0.2522$ & $\mathrm{R}^{2}=0.9950$ & $0.42-3.38$ \\
\hline DTBPH $^{m}$ & B15 & 2308.7 & B & 191 & 4-Ethylphenol & $y=166.76 x+11.394$ & $\mathrm{R}^{2}=0.9934$ & 25.00-100.00 \\
\hline Coumaran & B16 & 2403.1 & B & 120 & 4-Ethylphenol & $y=159.63 x+29.604$ & $\mathrm{R}^{2}=0.9862$ & $12.50-800.00$ \\
\hline
\end{tabular}


Table 2. Cont

\begin{tabular}{|c|c|c|c|c|c|c|c|c|}
\hline Volatile & $\mathrm{CN}^{\mathrm{a}}$ & RI & ID $^{b}$ & $Q^{c}$ & Standard & Quantitative Curve & $\mathbf{R}^{2}$ & Linearity Range $(\mu \mathrm{g} / \mathrm{L})$ \\
\hline \multicolumn{9}{|l|}{ Phenols } \\
\hline Ethyl salicylate & B17 & 1824.6 & $\mathrm{~A}$ & 120 & Ethyl salicylate & $y=60.837 x+1.9511$ & $\mathrm{R}^{2}=0.9906$ & $2.13-34.00$ \\
\hline 4-Ethylphenol & B18 & 2182.8 & A & 107 & 4-Ethylphenol & $y=162.82 x+18.57$ & $\mathrm{R}^{2}=0.9863$ & $1.56-800.00$ \\
\hline 4-Vinylguaiacol & B19 & 2208.5 & B & 135 & 4-Ethylphenol & $y=141.15 x+101.45$ & $\mathrm{R}^{2}=0.9973$ & $200.00-800.00$ \\
\hline$\frac{\text { Esters }}{\text { Ethyl esters }}$ & E & & & & & & & \\
\hline Ethyl Acetate & E1 & 764.8 & A & 43 & Ethyl Acetate & $y=6076.6 x-2518.1$ & $\mathrm{R}^{2}=0.9978$ & 19187.50-153500.00 \\
\hline Ethyl butanoate & E2 & 1037.5 & A & 71 & Ethyl butanoate & $y=613.77 x+1.0444$ & $\mathrm{R}^{2}=0.9999$ & $5.41-692.50$ \\
\hline Ethyl hexanoate & E3 & 1244.4 & $\mathrm{~A}$ & 88 & Ethyl hexanoate & $y=68.562 x-6.8083$ & $R^{2}=1$ & $40.63-650.00$ \\
\hline Ethyl 3-hexenoate & $\mathrm{E} 4$ & 1307.6 & $\mathrm{~A}$ & 69 & Ethyl 3-hexenoate & $y=80.813 x-0.0169$ & $\mathrm{R}^{2}=0.9976$ & $0.62-9.94$ \\
\hline Ethyl enanthate & E5 & 1337.6 & $\mathrm{~A}$ & 88 & Ethyl enanthate & $y=35.894 x+0.1203$ & $\mathrm{R}^{2}=0.9997$ & $0.91-3.63$ \\
\hline Ethyl lactate & E6 & 1346.8 & A & 45 & Ethyl lactate & $y=28150 x-92.569$ & $\mathrm{R}^{2}=0.9960$ & $199.22-6375.00$ \\
\hline Ethyl octanoate & E7 & 1438.7 & $\mathrm{~A}$ & 88 & Ethyl octanoate & $y=42.269 x+91.381$ & $\mathrm{R}^{2}=0.9998$ & $318.75-1275.00$ \\
\hline Ethyl mesitylacetate & E8 & 1511.8 & $\mathrm{~B}$ & 133 & Ethyl phenacetate & $y=38.305 x+0.1918$ & $\mathrm{R}^{2}=0.9779$ & $0.42-1.69$ \\
\hline Ethyl nonanoate & E9 & 1539.5 & $\mathrm{~A}$ & 88 & Ethyl nonanoate & $y=42.505 x+0.9106$ & $\mathrm{R}^{2}=0.9940$ & $1.50-12.03$ \\
\hline $\mathrm{E} 2 \mathrm{H} 4 \mathrm{MP}^{\mathrm{n}}$ & E10 & 1546.9 & $\mathrm{~A}$ & 69 & E2H4MP & $y=394.42 x+1.2915$ & $\mathrm{R}^{2}=0.9999$ & $8.87-35.47$ \\
\hline Ethyl caprate & E11 & 1642.8 & A & 88 & Ethyl caprate & $y=78.134 x+132.78$ & $\mathrm{R}^{2}=0.9911$ & $159.38-1275.00$ \\
\hline Ethyl benzoate & E12 & 1677.4 & $\mathrm{~B}$ & 105 & Ethyl phenacetate & $y=35.188 x+0.2362$ & $\mathrm{R}^{2}=0.9924$ & $0.84-3.38$ \\
\hline Ethyl 9-decenoate & E13 & 1694.3 & B & 55 & Ethyl laurate & $y=278.11 x+0.9429$ & $\mathrm{R}^{2}=0.9891$ & $0.65-41.64$ \\
\hline Ethyl laurate & E14 & 1847.5 & $\mathrm{~A}$ & 88 & Ethyl laurate & $y=97.913 x+53.207$ & $\mathrm{R}^{2}=0.9985$ & $83.28-333.13$ \\
\hline Ethyl myristate & E15 & 2053.2 & $\mathrm{~B}$ & 88 & Ethyl laurate & $y=255.61 x+3.5176$ & $\mathrm{R}^{2}=0.9965$ & $10.41-41.64$ \\
\hline Ethyl hexadecanoate & E16 & 2256.1 & B & 88 & Ethyl laurate & $y=140.86 x+16.882$ & $\mathrm{R}^{2}=0.9696$ & $10.41-166.56$ \\
\hline \multicolumn{9}{|l|}{ Acetates } \\
\hline Isobutyl acetate & E17 & 1009.6 & A & 43 & Isobutyl acetate & $y=417.2 x-134.5$ & $R^{2}=0.9996$ & $17.81-570.00$ \\
\hline Isopentyl acetate & E18 & 1135.9 & $\mathrm{~A}$ & 43 & Isopentyl acetate & $y=186.69 x-260.63$ & $\mathrm{R}^{2}=0.9983$ & $631.88-10110.00$ \\
\hline Hexyl acetate & E19 & 1298.8 & A & 43 & Hexyl acetate & $\mathrm{y}=44.969 x-0.3454$ & $\mathrm{R}^{2}=0.9999$ & $0.29-75.47$ \\
\hline Heptyl acetate & E20 & 1377.4 & B & 43 & Hexyl acetate & $y=46.025 x-0.4832$ & $\mathrm{R}^{2}=0.9999$ & $2.36-18.87$ \\
\hline 2-Ethyl-1-hexanol acetate & E21 & 1387.2 & B & 43 & 2-Heptanol & $y=224.82 x-0.2812$ & $\mathrm{R}^{2}=0.9985$ & $1.22-19.50$ \\
\hline Octyl acetate & E22 & 1477 & B & 43 & 2-Heptanol & $y=224.82 x-0.2812$ & $\mathrm{R}^{2}=0.9985$ & $1.22-19.50$ \\
\hline Trimethylene acetate & E23 & 1740.3 & B & 43 & Diethyl succinate & $y=749.61 x+12.249$ & $\mathrm{R}^{2}=0.9987$ & $38.67-309.38$ \\
\hline
\end{tabular}


Table 2. Cont

\begin{tabular}{|c|c|c|c|c|c|c|c|c|}
\hline Volatile & $\mathrm{CN}^{\mathrm{a}}$ & RI & ID $^{b}$ & $\mathrm{QI}^{\mathrm{c}}$ & Standard & Quantitative Curve & $\mathbf{R}^{2}$ & Linearity Range $(\mu \mathrm{g} / \mathrm{L})$ \\
\hline \multicolumn{9}{|l|}{ Other esters } \\
\hline Isobutyl hexanoate & E24 & 1357.7 & B & 99 & Styrene & $y=54.92 x-0.4452$ & $R^{2}=0.9928$ & $0.25-3.99$ \\
\hline Methyl octanoate & E25 & 1393.8 & $\mathrm{~A}$ & 74 & Methyl octanoate & $y=23.885 x+0.7001$ & $\mathrm{R}^{2}=0.9991$ & $0.76-48.75$ \\
\hline Isopentyl hexanoate & E26 & 1462.3 & $\mathrm{~A}$ & 70 & Isopentyl hexanoate & $y=58.981 x+0.1264$ & $\mathrm{R}^{2}=0.9945$ & $0.61-9.77$ \\
\hline Isobutyl octanoate & E27 & 1554.6 & $\mathrm{~B}$ & 57 & Isoamyl caprylate & $y=33.7 x+0.8987$ & $\mathrm{R}^{2}=0.9878$ & $0.63-10.13$ \\
\hline Methyl caprate & E28 & 1599.0 & $\mathrm{~B}$ & 74 & Ethyl caprate & $y=277.88 x-4.4049$ & $\mathrm{R}^{2}=0.9917$ & $4.98-39.84$ \\
\hline Isoamyl octanoate & E29 & 1662.7 & $\mathrm{~A}$ & 70 & Isoamyl octanoate & $y=25.546 x+1.5448$ & $\mathrm{R}^{2}=0.9815$ & $0.63-20.25$ \\
\hline Diethyl succinate & E30 & 1680.1 & $\mathrm{~A}$ & 101 & Diethyl succinate & $y=709.47 x+17.935$ & $\mathrm{R}^{2}=0.9991$ & $38.67-618.75$ \\
\hline Isoamyl decanoate & E31 & 1866.8 & $\mathrm{~B}$ & 70 & Isoamyl caprylate & $y=32.527 x+1.1504$ & $\mathrm{R}^{2}=0.9920$ & $1.27-10.13$ \\
\hline $\mathrm{IMDMMP}^{\mathrm{o}}$ & E32 & 1883.2 & $\mathrm{~B}$ & 71 & Isoamyl caprylate & $y=25.382 x+1.5173$ & $\mathrm{R}^{2}=0.9829$ & $0.63-20.25$ \\
\hline NA3MBE $\mathrm{P}$ & E33 & 1905.7 & $\mathrm{~B}$ & 141 & Isoamyl caprylate & $y=32.527 x+1.1504$ & $\mathrm{R}^{2}=0.9920$ & $1.27-10.13$ \\
\hline Others & $\mathrm{O}$ & & & & & & & \\
\hline$\overline{\text { Styrene }}$ & O1 & 1269.8 & $\mathrm{~A}$ & 104 & Styrene & $y=37.916 x+1.5324$ & $R^{2}=0.9989$ & 31.94-127.75 \\
\hline$\alpha$-Ionene & $\mathrm{O} 2$ & 1489.7 & B & 159 & Ethyl salicylate & $y=60.837 x+1.9511$ & $\mathrm{R}^{2}=0.9906$ & $2.13-34.00$ \\
\hline ODETM q & $\mathrm{O} 3$ & 1549.5 & $\mathrm{~B}$ & 138 & Ethyl salicylate & $y=78.368 x+0.3169$ & $\mathrm{R}^{2}=0.9952$ & $1.06-17.00$ \\
\hline$\alpha$-Cedrene & $\mathrm{O} 4$ & 1625.9 & B & 119 & E-Nerolidol & $y=12.967 x+0.0836$ & $R^{2}=0.9864$ & $0.03-2.13$ \\
\hline$\alpha$-Calacorene & O5 & 1931.9 & B & 157 & Ethyl salicylate & $y=76.261 x+0.6681$ & $\mathrm{R}^{2}=0.9980$ & $2.13-17.00$ \\
\hline$\beta$-Ionone & O6 & 1951.7 & B & 177 & Ethyl salicylate & $y=78.368 x+0.3169$ & $\mathrm{R}^{2}=0.9952$ & $1.06-17.00$ \\
\hline DMUDL $^{r}$ & O7 & 1955.5 & B & 109 & E-Nerolidol & $y=16.415 x+0.0339$ & $\mathrm{R}^{2}=0.9952$ & $0.07-1.06$ \\
\hline Cedrol & O8 & 2137.5 & B & 95 & E-Nerolidol & $y=15.972 x+0.0556$ & $\mathrm{R}^{2}=0.9981$ & $0.13-1.06$ \\
\hline
\end{tabular}

a Code name. ${ }^{\mathrm{b}}$ Identification of the compounds: " $\mathrm{A}$ " means compound identified by mass spectrum and RI according to the standard, whereas " $\mathrm{B}$ " represents compound tentatively identified by mass spectrum with database and RI according to literatures. ${ }^{c}$ Quantitation ion. ${ }^{\mathrm{d}} 1,2-$-Dimethyl-cyclopentene-2-enecarboxylic acid.

e 2,2,6-Trimethylcyclohexanone. ${ }^{\mathrm{f}}$ 6-Methyl-5-heptene-2-one. ${ }^{\mathrm{g}}\left(2,6,6\right.$-Trimethyl-2-hydroxycyclohexylidene)acetic acid lactone. ${ }^{\mathrm{h}}$ 1,1,4,6-Tetramethylindane. ${ }^{\mathrm{i}}$ 2,5-Diisopropyl-p-xylene.

j 2,5,8-Trimethyl-1,2-dihydronaphthalene. ${ }^{\mathrm{k}}$ Methyl 2-(pentyloxy)benzoate. ${ }^{1}$ 3-Phenyl-4-hydroxyacetophenone. ${ }^{\mathrm{m}}$ 2,4-Di-tert-butylphenol. ${ }^{\mathrm{n}}$ Ethyl 2-hydroxy-4-methylpentanoate.

o 1-[2-(Isobutyryloxy)-1-methylethyl]-2,2-dimethylpropyl 2-methylpropanoate. P Nonanoic acid, 3-methylbutyl-2 ester. q 1-Oxaspiro[4.5]dec-6-ene, 2,6,10,10-tetramethyl-.

${ }^{r}$ 2,6-Dimethyl-2,6-undecadien-10-ol. 
Table 3. Concentration of volatile compounds in goji berry fermented wine.

\begin{tabular}{|c|c|c|c|c|c|c|c|c|}
\hline Volatile $(\mu \mathrm{g} / \mathrm{L})$ & DF & DF-G & DP & DP-G & FF & FF-G & FP & FP-G \\
\hline \multicolumn{9}{|l|}{ Volatile acids } \\
\hline DMECAd & $40.8 \pm 1.5 \mathrm{a}^{*}$ & $41.2 \pm 1.0 \mathrm{a}$ & $39.8 \pm 0.7 \mathrm{a}$ & $41.3 \pm 1.4 \mathrm{a}$ & $2.5 \pm 0.0 \mathrm{~b}$ & $2.5 \pm 0.1 \mathrm{~b}$ & $2.6 \pm 0.2 \mathrm{~b}$ & $2.4 \pm 0.1 \mathrm{~b}$ \\
\hline Acetic acid & $172.6 \pm 53.0 \mathrm{bc}$ & $199.8 \pm 96.6 \mathrm{bc}$ & $135.5 \pm 81.7 \mathrm{c}$ & $177.3 \pm 32.0 \mathrm{bc}$ & $469.4 \pm 122.5 \mathrm{abc}$ & $417.6 \pm 86.1 \mathrm{abc}$ & $639.3 \pm 121.1 \mathrm{a}$ & $525.3 \pm 101.0 \mathrm{ab}$ \\
\hline Butanoic acid & $1506.8 \pm 135.7 \mathrm{a}$ & $1489.4 \pm 359.1 \mathrm{a}$ & $1488.7 \pm 213.3 \mathrm{a}$ & $1503.4 \pm 69.9 \mathrm{a}$ & $414.3 \pm 99.4 \mathrm{~b}$ & $412.4 \pm 13.0 \mathrm{~b}$ & $418.2 \pm 71.9 \mathrm{~b}$ & $373.7 \pm 5.8 \mathrm{~b}$ \\
\hline$\alpha$-Methylbutyric acid & $1532.2 \pm 42.6 \mathrm{a}$ & $1450.2 \pm 188.3 \mathrm{a}$ & $1544.0 \pm 210.31 \mathrm{a}$ & $1519.5 \pm 14.8 \mathrm{a}$ & $531.2 \pm 104.0 \mathrm{~b}$ & $488.5 \pm 6.4 \mathrm{~b}$ & $539.4 \pm 9.3 \mathrm{~b}$ & $473.4 \pm 5.3 \mathrm{~b}$ \\
\hline Octanoic acid & $1018.7 \pm 49.8 \mathrm{a}$ & $819.8 \pm 297.4 \mathrm{a}$ & $1045.5 \pm 122.6 \mathrm{a}$ & $914.2 \pm 10.2 \mathrm{a}$ & $740.8 \pm 318.8 \mathrm{a}$ & $598.5 \pm 172.9 \mathrm{a}$ & $610.5 \pm 39.9 \mathrm{a}$ & $563.6 \pm 38.0 \mathrm{a}$ \\
\hline Decanoic acid & $2712.4 \pm 146.2 \mathrm{a}$ & $2186.8 \pm 995.4 \mathrm{a}$ & $6053.6 \pm 1901.4 \mathrm{a}$ & $3325.7 \pm 203.0 \mathrm{a}$ & $5047.2 \pm 1410.4 \mathrm{a}$ & $4215.8 \pm 1303.4 \mathrm{a}$ & $2570.7 \pm 28.2 \mathrm{a}$ & $2752.2 \pm 385.4 \mathrm{a}$ \\
\hline Total & $6983.5 \pm 340.5 a b$ & $6187.3 \pm 1936.0 a b$ & $10306.8 \pm 1274.1 a$ & $7481.4 \pm 98.0 a b$ & $7205.3 \pm 2055.1 a b$ & $6135.4 \pm 1568.9 a b$ & $4780.6 \pm 195.3 \mathrm{~b}$ & $4690.6 \pm 235.5 b$ \\
\hline \multicolumn{9}{|l|}{ Higher alcohols } \\
\hline 2-Methyl-1-propanol & $35548.7 \pm 1625.3 \mathrm{a}$ & $35130.6 \pm 589.8 \mathrm{a}$ & $36260.1 \pm 1605.77 a$ & $35318.8 \pm 1043.7 \mathrm{a}$ & $23463.5 \pm 907.0 \mathrm{~b}$ & $23601.0 \pm 687.37 \mathrm{~b}$ & $20073.4 \pm 98.2 \mathrm{~b}$ & $20012.0 \pm 418.5 \mathrm{~b}$ \\
\hline 1-Butanol & $967.2 \pm 87.1 \mathrm{a}$ & $955.0 \pm 104.7 \mathrm{a}$ & $948.5 \pm 42.7 \mathrm{a}$ & $953.4 \pm 10.5 \mathrm{a}$ & $948.0 \pm 33.48 \mathrm{a}$ & $978.0 \pm 61.4 \mathrm{a}$ & $929.3 \pm 103.8 \mathrm{a}$ & $866.2 \pm 16.9 \mathrm{a}$ \\
\hline Isopentyl alcohol & $187579.3 \pm 2742.2 \mathrm{~b}$ & $184874.0 \pm 6446.52 \mathrm{~b}$ & $188753.9 \pm 5009.5 b$ & $183698.5 \pm 816.3 \mathrm{~b}$ & $217389.5 \pm 7670.1 \mathrm{a}$ & $214993.6 \pm 267.8 \mathrm{a}$ & $173630.3 \pm 1822.6 \mathrm{~b}$ & $173019.9 \pm 48.9 b$ \\
\hline Pentyl alcohol & $17.0 \pm 3.19 c$ & $21.4 \pm 3.1 \mathrm{bc}$ & $17.9 \pm 3.3 \mathrm{c}$ & $15.7 \pm 0.3 c$ & $28.1 \pm 1.1 \mathrm{ab}$ & $27.5 \pm 3.0 \mathrm{ab}$ & $32.1 \pm 0.2 \mathrm{a}$ & $34.3 \pm 1.8 \mathrm{a}$ \\
\hline 1-Hexanol & $91.5 \pm 3.0 \mathrm{~b}$ & $99.3 \pm 5.3 \mathrm{~b}$ & $92.8 \pm 6.5 \mathrm{~b}$ & $90.5 \pm 5.1 \mathrm{~b}$ & $94.5 \pm 2.7 \mathrm{~b}$ & $101.5 \pm 1.7 \mathrm{~b}$ & $160.7 \pm 1.0 \mathrm{a}$ & $169.1 \pm 2.7 \mathrm{a}$ \\
\hline (Z)-3-Hexen-1-ol & $123.8 \pm 21.6 \mathrm{a}$ & $132.7 \pm 5.2 \mathrm{a}$ & $134.0 \pm 4.58 \mathrm{a}$ & $115.6 \pm 8.4 \mathrm{a}$ & $100.3 \pm 0.4 \mathrm{a}$ & $122.4 \pm 21.7 \mathrm{a}$ & $129.8 \pm 32.3 \mathrm{a}$ & $144.4 \pm 29.2 \mathrm{a}$ \\
\hline 2-Heptanol & $5.6 \pm 0.1 \mathrm{c}$ & $5.7 \pm 0.3 \mathrm{c}$ & $6.3 \pm 0.2 \mathrm{bc}$ & $6.3 \pm 0.1 \mathrm{bc}$ & $7.8 \pm 0.3 \mathrm{a}$ & $7.3 \pm 0.1 \mathrm{ab}$ & $6.7 \pm 0.1 \mathrm{abc}$ & $7.8 \pm 0.6 \mathrm{a}$ \\
\hline 3-Ethoxy-1-propanol & $39.9 \pm 1.6 \mathrm{a}$ & $35.1 \pm 15.8 \mathrm{a}$ & $33.7 \pm 9.1 \mathrm{a}$ & $32.6 \pm 1.1 \mathrm{a}$ & $32.5 \pm 3.1 \mathrm{a}$ & $26.4 \pm 6.1 \mathrm{a}$ & $34.9 \pm 2.9 \mathrm{a}$ & $32.4 \pm 3.8 \mathrm{a}$ \\
\hline 1-Octen-3-ol & $6.8 \pm 0.6 \mathrm{a}$ & $7.6 \pm 0.1 \mathrm{a}$ & $6.9 \pm 0.3 \mathrm{a}$ & $8.0 \pm 0.56 a$ & $9.4 \pm 3.8 \mathrm{a}$ & $13.6 \pm 0.1 \mathrm{a}$ & $13.9 \pm 0.5 \mathrm{a}$ & $13.2 \pm 8.1 \mathrm{a}$ \\
\hline Sulcatol & $\mathrm{TR}^{* *}$ & $\mathrm{TR}$ & TR & TR & TR & TR & TR & TR \\
\hline 2-Ethyl-1-hexanol & $12.2 \pm 0.2 \mathrm{f}$ & $13.0 \pm 0.4 \mathrm{ef}$ & $16.2 \pm 1.2 \mathrm{~cd}$ & $15.4 \pm 0.3 \mathrm{de}$ & $19.4 \pm 1.4 \mathrm{~b}$ & $18.4 \pm 0.2 \mathrm{bc}$ & $75.5 \pm 0.5 \mathrm{a}$ & $76.0 \pm 0.2 \mathrm{a}$ \\
\hline$\left[R-\left(R^{*}, R^{*}\right)\right]-2,3$-Butanediol & $375898.6 \pm 82505.1 \mathrm{a}$ & $363649.8 \pm 241292.74 \mathrm{a}$ & $348050.8 \pm 196950.2 \mathrm{a}$ & $279674.0 \pm 61987.2 \mathrm{a}$ & $1766887.6 \pm 1645173.9 \mathrm{a}$ & $979257.2 \pm 660565.6 \mathrm{a}$ & $1313477.4 \pm 699634.8 \mathrm{a}$ & $1735789.3 \pm 233651.6 \mathrm{a}$ \\
\hline 1-Octanol & $10.9 \pm 2.9 \mathrm{~b}$ & $11.1 \pm 2.0 \mathrm{~b}$ & $9.3 \pm 2.1 \mathrm{~b}$ & $10.2 \pm 1.2 \mathrm{~b}$ & $12.3 \pm 0.8 \mathrm{~b}$ & $11.8 \pm 0.5 \mathrm{~b}$ & $31.9 \pm 0.7 \mathrm{a}$ & $33.1 \pm 0.5 \mathrm{a}$ \\
\hline$\left[S-\left(R^{*}, R^{*}\right)\right]$-2,3-Butanediol & $117488.4 \pm 16482.5 \mathrm{a}$ & $123910.7 \pm 67104.1 \mathrm{a}$ & $124577.5 \pm 48004.6 \mathrm{a}$ & $86202.4 \pm 13306.7 \mathrm{a}$ & $305149.2 \pm 252625.20 \mathrm{a}$ & $190949.9 \pm 107619.4 \mathrm{a}$ & $205124.8 \pm 82211.3 \mathrm{a}$ & $338514.7 \pm 20924.2 \mathrm{a}$ \\
\hline trans-2-Octenol & $9.5 \pm 0.7 \mathrm{~b}$ & $10.3 \pm 0.4 \mathrm{~b}$ & $9.5 \pm 0.3 \mathrm{~b}$ & $10.0 \pm 1.21 \mathrm{~b}$ & $5.3 \pm 0.4 \mathrm{a}$ & $5.0 \pm 0.1 \mathrm{a}$ & $4.6 \pm 0.0 \mathrm{a}$ & $5.9 \pm 0.5 \mathrm{a}$ \\
\hline 1-Nonanol & $0.1 \pm 0.0 \mathrm{c}$ & $0.1 \pm 0.0 \mathrm{c}$ & $0.1 \pm 0.0 \mathrm{c}$ & $0.1 \pm 0.0 \mathrm{c}$ & $0.2 \pm 0.0 \mathrm{~b}$ & $0.2 \pm 0.0 \mathrm{bc}$ & $0.3 \pm 0.0 \mathrm{a}$ & $0.3 \pm 0.0 \mathrm{a}$ \\
\hline Methionol & $1316.9 \pm 37.0 \mathrm{a}$ & $1162.2 \pm 63.3 \mathrm{a}$ & $1276.4 \pm 540.2 \mathrm{a}$ & $1280.3 \pm 143.6 \mathrm{a}$ & $775.5 \pm 483.1 \mathrm{a}$ & $544.1 \pm 340.8 \mathrm{a}$ & $816.2 \pm 254.5 \mathrm{a}$ & $770.5 \pm 168.4 \mathrm{a}$ \\
\hline$E$-5-Decen-1-ol & $N D^{* * *}$ & ND & ND & $\mathrm{ND}$ & $8.9 \pm 1.7 \mathrm{~b}$ & $7.7 \pm 0.8 \mathrm{~b}$ & $61.7 \pm 1.2 \mathrm{a}$ & $58.8 \pm 1.1 \mathrm{a}$ \\
\hline Phenylethyl Alcohol & $20978.7 \pm 1150.9 \mathrm{a}$ & $20111.8 \pm 5434.5 \mathrm{a}$ & $19807.4 \pm 7958.7 \mathrm{a}$ & $20117.5 \pm 489.0 \mathrm{a}$ & $15526.1 \pm 8671.7 \mathrm{a}$ & $11607.3 \pm 4687.1 \mathrm{a}$ & $14267.9 \pm 1231.0 \mathrm{a}$ & $13133.9 \pm 2106.6 \mathrm{a}$ \\
\hline Total & $740054.1 \pm 98959.9 a$ & $730092.8 \pm 320914.5 a$ & $719960.1 \pm 246869.2 a$ & $607507.9 \pm 77776.8 a$ & $2330406.2 \pm 1915574.3 a$ & $1422220.4 \pm 773718.3 a$ & $1728818.3 \pm 781475.9 a$ & $2282631.4 \pm 257372.4 a$ \\
\hline \multicolumn{9}{|l|}{ Aldehyde/Ketones } \\
\hline Nonanal & $14.2 \pm 0.3 \mathrm{a}$ & $15.7 \pm 1.2 \mathrm{a}$ & $10.7 \pm 0.7 \mathrm{a}$ & $12.8 \pm 0.0 \mathrm{a}$ & $5.8 \pm 0.3 \mathrm{a}$ & $5.1 \pm 0.3 \mathrm{a}$ & $4.5 \pm 0.3 \mathrm{a}$ & $4.5 \pm 0.9 \mathrm{a}$ \\
\hline Furfural & $492.2 \pm 0.8 \mathrm{a}$ & $484.6 \pm 32.0 \mathrm{a}$ & $436.9 \pm 38.4 \mathrm{a}$ & $465.3 \pm 4.0 \mathrm{a}$ & $11.7 \pm 2.9 \mathrm{~b}$ & $11.7 \pm 3.3 \mathrm{~b}$ & $12.8 \pm 1.6 \mathrm{~b}$ & $11.8 \pm 0.3 \mathrm{~b}$ \\
\hline Decanal & $3.7 \pm 0.7 \mathrm{a}$ & $4.9 \pm 1.0 \mathrm{a}$ & $2.5 \pm 0.0 \mathrm{a}$ & $4.5 \pm 0.6 \mathrm{a}$ & $4.0 \pm 0.90 \mathrm{a}$ & $2.4 \pm 0.3 \mathrm{a}$ & $2.4 \pm 0.6 \mathrm{a}$ & $3.7 \pm 1.2 \mathrm{a}$ \\
\hline Safranal & $2.3 \pm 0.0 \mathrm{a}$ & $2.4 \pm 0.2 \mathrm{a}$ & $2.2 \pm 0.1 \mathrm{a}$ & $2.2 \pm 0.1 \mathrm{a}$ & $0.5 \pm 0.0 \mathrm{~b}$ & $0.5 \pm 0.0 \mathrm{~b}$ & $0.6 \pm 0.0 \mathrm{~b}$ & $0.6 \pm 0.0 \mathrm{~b}$ \\
\hline 2-Butyl-2-octenal & $16.7 \pm 1.3 \mathrm{a}$ & $17.1 \pm 3.1 \mathrm{a}$ & $17.4 \pm 3.4 \mathrm{a}$ & $18.1 \pm 0.4 \mathrm{a}$ & $11.9 \pm 2.4 \mathrm{a}$ & $10.6 \pm 1.4 \mathrm{a}$ & $11.0 \pm 1.1 \mathrm{a}$ & $10.9 \pm 1.3 \mathrm{a}$ \\
\hline Isobutyl ketone & $22.0 \pm 5.0 \mathrm{ab}$ & $24.0 \pm 8.0 \mathrm{ab}$ & $7.58 \pm 5.93 \mathrm{ab}$ & $33.1 \pm 13.2 \mathrm{a}$ & TR & $2.48 \pm 0.37 \mathrm{~b}$ & TR & $0.09 \pm 0.53 b$ \\
\hline TMCHN & $\mathrm{TR}$ & TR & TR & TR & $\mathrm{TR}$ & $\mathrm{TR}$ & $\mathrm{TR}$ & $\mathrm{TR}$ \\
\hline 6M5H2NE & $11.0 \pm 1.15 \mathrm{a}$ & $11.9 \pm 4.1 \mathrm{a}$ & $8.2 \pm 1.1 \mathrm{a}$ & $8.0 \pm 0.7 \mathrm{a}$ & $\mathrm{TR}$ & $\mathrm{TR}$ & TR & TR \\
\hline 2-Undecanone & $1.9 \pm 0.0 \mathrm{~b}$ & $2.1 \pm 0.1 \mathrm{~b}$ & $2.0 \pm 0.1 \mathrm{a}$ & $2.0 \pm 0.07 \mathrm{a}$ & $3.5 \pm 0.5 c$ & $3.2 \pm 0.1 \mathrm{c}$ & $3.6 \pm 0.0 \mathrm{~d}$ & $3.9 \pm 0.2 \mathrm{~cd}$ \\
\hline$\beta$-Cyclocitral & $6.5 \pm 0.1 \mathrm{a}$ & $6.6 \pm 0.4 \mathrm{a}$ & $5.6 \pm 0.0 \mathrm{~b}$ & $5.6 \pm 0.1 \mathrm{~b}$ & $3.0 \pm 0.2 \mathrm{c}$ & $3.4 \pm 0.3 \mathrm{c}$ & $5.3 \pm 0.1 \mathrm{~b}$ & $4.9 \pm 0.1 \mathrm{~b}$ \\
\hline Dihydropseudoionone & $4.4 \pm 0.4 \mathrm{ab}$ & $4.5 \pm 0.1 \mathrm{ab}$ & $5.5 \pm 2.3 \mathrm{a}$ & $3.7 \pm 0.1 \mathrm{abc}$ & $1.3 \pm 0.3 \mathrm{~cd}$ & $0.9 \pm 0.1 \mathrm{c}$ & $2.7 \pm 0.0 \mathrm{abc}$ & $2.8 \pm 0.1 \mathrm{abc}$ \\
\hline 2-Acetylpyrrole & $121.6 \pm 8.1 \mathrm{a}$ & $116.16 \pm 43.2 \mathrm{a}$ & $114.3 \pm 53.6 \mathrm{a}$ & $111.7 \pm 0.5 \mathrm{a}$ & ND & ND & ND & ND \\
\hline TMHAAL & $6.8 \pm 0.34 \mathrm{a}$ & $6.5 \pm 1.6 \mathrm{a}$ & $7.1 \pm 1.6 \mathrm{a}$ & $6.0 \pm 0.45 \mathrm{a}$ & $0.9 \pm 0.1 \mathrm{~b}$ & $1.6 \pm 0.5 b$ & $1.4 \pm 0.1 \mathrm{~b}$ & $1.1 \pm 0.1 \mathrm{~b}$ \\
\hline Total & $702.1 \pm 13.8 a$ & $696.3 \pm 70.2 a$ & $618.8 \pm 100.2 b$ & $671.7 \pm 8.3 a b$ & $37.4 \pm 7.0 c$ & $37.4 \pm 6.0 c$ & $42.3 \pm 0.0 c$ & $43.4 \pm 1.9 c$ \\
\hline
\end{tabular}


Table 3. Cont.

\begin{tabular}{|c|c|c|c|c|c|c|c|c|}
\hline Volatile $(\mu \mathrm{g} / \mathrm{L})$ & DF & DF-G & DP & DP-G & FF & FF-G & FP & FP-G \\
\hline \multicolumn{9}{|l|}{ Benzene } \\
\hline Isodurene & $4.3 \pm 0.1 \mathrm{a}$ & $4.1 \pm 0.2 \mathrm{a}$ & $4.6 \pm 0.2 \mathrm{a}$ & $4.6 \pm 0.4 \mathrm{a}$ & $1.3 \pm 0.0 \mathrm{~b}$ & $1.3 \pm 0.1 \mathrm{~b}$ & $1.0 \pm 0.1 \mathrm{~b}$ & $0.9 \pm 0.0 \mathrm{~b}$ \\
\hline TEMLNE & $7.2 \pm 0.1 \mathrm{a}$ & $5.8 \pm 1.4 \mathrm{abc}$ & $5.8 \pm 2.1 \mathrm{abc}$ & $6.9 \pm 0.7 \mathrm{ab}$ & $2.8 \pm 0.6 \mathrm{c}$ & $2.2 \pm 0.2 \mathrm{c}$ & $3.8 \pm 0.1 \mathrm{abc}$ & $3.1 \pm 0.7 \mathrm{bc}$ \\
\hline DPPXL & $31.1 \pm 0.9 \mathrm{a}$ & $29.2 \pm 1.3 \mathrm{ab}$ & $24.3 \pm 7.7 \mathrm{abc}$ & $28.4 \pm 2.0 \mathrm{ab}$ & $18.4 \pm 1.5 \mathrm{bc}$ & $18.2 \pm 0.1 \mathrm{bc}$ & $16.7 \pm 0.6 c$ & $16.5 \pm 0.6 c$ \\
\hline Naphthalene & $4.5 \pm 0.2 \mathrm{a}$ & $5.3 \pm 0.4 \mathrm{a}$ & $4.5 \pm 0.3 \mathrm{a}$ & $4.7 \pm 0.1 \mathrm{a}$ & $2.2 \pm 0.3 \mathrm{~b}$ & $2.0 \pm 0.1 \mathrm{~b}$ & $2.3 \pm 0.0 \mathrm{~b}$ & $2.3 \pm 0.0 \mathrm{~b}$ \\
\hline TMDHPE & $6.2 \pm 0.2 \mathrm{a}$ & $5.9 \pm 0.1 \mathrm{a}$ & $5.3 \pm 1.1 \mathrm{a}$ & $5.8 \pm 0.5 \mathrm{a}$ & $1.9 \pm 0.2 \mathrm{~b}$ & $1.6 \pm 0.0 \mathrm{~b}$ & $2.4 \pm 0.2 \mathrm{~b}$ & $2.4 \pm 0.3 \mathrm{~b}$ \\
\hline $\mathrm{M} 2 \mathrm{~PB}$ & $1.5 \pm 0.1 \mathrm{ab}$ & $1.6 \pm 0.0 \mathrm{ab}$ & $1.5 \pm 0.1 \mathrm{ab}$ & $1.3 \pm 0.0 \mathrm{~b}$ & $1.9 \pm 0.3 \mathrm{a}$ & $1.7 \pm 0.0 \mathrm{ab}$ & $1.7 \pm 0.0 \mathrm{ab}$ & $1.7 \pm 0.1 \mathrm{ab}$ \\
\hline Phenethyl acetate & $144.7 \pm 10.9 \mathrm{a}$ & $141.0 \pm 17.4 \mathrm{a}$ & $114.9 \pm 15.3 \mathrm{a}$ & $122.2 \pm 1.0 \mathrm{a}$ & $43.6 \pm 7.4 \mathrm{~b}$ & $37.5 \pm 2.1 \mathrm{~b}$ & $28.1 \pm 0.7 b$ & $26.4 \pm 0.2 \mathrm{~b}$ \\
\hline$\beta$-Methylnaphthalene & $17.2 \pm 0.4 \mathrm{a}$ & $16.0 \pm 1.6 \mathrm{a}$ & $16.0 \pm 1.8 \mathrm{a}$ & $17.0 \pm 0.44 \mathrm{a}$ & $5.6 \pm 0.7 \mathrm{~b}$ & $5.4 \pm 0.1 \mathrm{~b}$ & $4.7 \pm 0.1 \mathrm{~b}$ & $4.7 \pm 0.1 \mathrm{~b}$ \\
\hline Benzyl alcohol & $308.3 \pm 2.0 \mathrm{a}$ & $297.9 \pm 60.4 \mathrm{a}$ & $305.6 \pm 111.7$ a & $314.6 \pm 7.7 \mathrm{a}$ & $314.5 \pm 140.8 \mathrm{a}$ & $289.1 \pm 75.4 \mathrm{a}$ & $527.1 \pm 23.0 \mathrm{a}$ & $514.1 \pm 80.8 \mathrm{a}$ \\
\hline Ethyl dihydrocinnamate & $1.2 \pm 0.0 \mathrm{~b}$ & $1.3 \pm 0.1 \mathrm{~b}$ & $1.2 \pm 0.0 \mathrm{~b}$ & $1.3 \pm 0.0 \mathrm{~b}$ & $3.2 \pm 0.6 \mathrm{a}$ & $2.7 \pm 0.2 \mathrm{a}$ & $3.2 \pm 0.1 \mathrm{a}$ & $3.1 \pm 0.1 \mathrm{a}$ \\
\hline$\alpha$-Methylnaphthalene & $11.4 \pm 0.2 \mathrm{a}$ & $10.8 \pm 0.7 \mathrm{a}$ & $11.1 \pm 1.1 \mathrm{a}$ & $11.5 \pm 0.3 \mathrm{a}$ & $2.7 \pm 0.5 \mathrm{~b}$ & $2.1 \pm 0.1 \mathrm{~b}$ & $2.0 \pm 0.1 \mathrm{~b}$ & $1.9 \pm 0.1 \mathrm{~b}$ \\
\hline 4-Tolylcarbinol & $1.4 \pm 0.1 \mathrm{a}$ & $1.3 \pm 0.3 \mathrm{a}$ & $1.4 \pm 0.1 \mathrm{a}$ & $1.4 \pm 0.1 \mathrm{a}$ & $0.9 \pm 0.9$ & $0.5 \pm 0.1$ & $1.0 \pm 0.06$ & $1.0 \pm 0.0$ \\
\hline Ethyl cinnamate & $1.9 \pm 0.2 \mathrm{a}$ & $2.5 \pm 0.5 \mathrm{a}$ & $2.4 \pm 0.4 \mathrm{a}$ & $2.9 \pm 0.1 \mathrm{a}$ & $2.1 \pm 0.4 \mathrm{a}$ & $2.5 \pm 0.3 \mathrm{a}$ & $1.8 \pm 0.0 \mathrm{a}$ & $2.2 \pm 0.1 \mathrm{a}$ \\
\hline $3 \mathrm{P} 4 \mathrm{HP}$ & $1.9 \pm 0.0 \mathrm{a}$ & $1.9 \pm 0.1 \mathrm{a}$ & $1.1 \pm 0.1 \mathrm{~b}$ & $1.2 \pm 0.1 \mathrm{~b}$ & $\mathrm{ND}$ & ND & ND & ND \\
\hline DTBPHm & $41.5 \pm 5.0 \mathrm{a}$ & $46.0 \pm 0.9 \mathrm{a}$ & $44.0 \pm 0.7 \mathrm{a}$ & $47.0 \pm 1.0 \mathrm{a}$ & $49.2 \pm 9.7 \mathrm{a}$ & $38.7 \pm 4.8 \mathrm{a}$ & $39.2 \pm 2.6 \mathrm{a}$ & $40.8 \pm 1.3 \mathrm{a}$ \\
\hline Coumaran & $202.5 \pm 4.54 \mathrm{a}$ & $196.6 \pm 43.0 \mathrm{a}$ & $240.6 \pm 66.7 \mathrm{a}$ & $219.0 \pm 3.9 \mathrm{a}$ & $1096.6 \pm 651.1 \mathrm{a}$ & $622.4 \pm 312.0 \mathrm{a}$ & $970.0 \pm 132.30 \mathrm{a}$ & $981.9 \pm 22.0 \mathrm{a}$ \\
\hline Ethyl salicylate & $6.1 \pm 0.97 \mathrm{~b}$ & $11.8 \pm 3.6 \mathrm{a}$ & $4.3 \pm 0.3 \mathrm{~b}$ & $4.8 \pm 0.3 \mathrm{~b}$ & $5.8 \pm 0.7 \mathrm{~b}$ & $5.3 \pm 0.3 \mathrm{~b}$ & $5.3 \pm 0.1 \mathrm{~b}$ & $5.6 \pm 0.0 \mathrm{~b}$ \\
\hline 4-Ethylphenol & $1466.5 \pm 3.1 \mathrm{~b}$ & $1480.8 \pm 5.3 \mathrm{~b}$ & $1482.8 \pm 14.6 \mathrm{~b}$ & $1464.6 \pm 0.9 \mathrm{~b}$ & $8167.3 \pm 3706.2 \mathrm{a}$ & $6012.7 \pm 1923.9 \mathrm{ab}$ & $11677.1 \pm 140.4 \mathrm{a}$ & $9968.4 \pm 1214.6 \mathrm{a}$ \\
\hline 4-Vinylguaiacol & $329.1 \pm 9.4 \mathrm{a}$ & $310.5 \pm 56.6 \mathrm{a}$ & $372.2 \pm 93.1 \mathrm{a}$ & $371.6 \pm 4.6 \mathrm{a}$ & $791.7 \pm 394.5 \mathrm{a}$ & $548.7 \pm 211.3 \mathrm{a}$ & $\begin{array}{r}656.6 \pm 15.3 \mathrm{a} \\
\text { a }\end{array}$ & $617.2 \pm 60.1 \mathrm{a}$ \\
\hline Total & $2588.3 \pm 23.0 \mathrm{~b}$ & $2570.2 \pm 185.4 b$ & $2643.6 \pm 285.7 b$ & $2630.6 \pm 23.2 b$ & $10511.8 \pm 4916.4 a b$ & $7594.7 \pm 2530.2 a b$ & $13943.7 \pm 312.4 a$ & $12194.2 \pm 1375.9 a$ \\
\hline \multicolumn{9}{|l|}{ Esters } \\
\hline Ethyl Acetate & $74687.2 \pm 1416.4 \mathrm{a}$ & $78072.4 \pm 2474.7 \mathrm{a}$ & $75663.3 \pm 6509.8 \mathrm{a}$ & $72540.3 \pm 646.6 \mathrm{a}$ & $47864.3 \pm 1550.9 \mathrm{~b}$ & $49750.7 \pm 3003.3 \mathrm{~b}$ & $52201.6 \pm 1608.2 \mathrm{~b}$ & $57245.4 \pm 1761.9 \mathrm{~b}$ \\
\hline Ethyl butanoate & $306.4 \pm 15.3 \mathrm{a}$ & $315.7 \pm 17.8 \mathrm{a}$ & $306.2 \pm 23.2 \mathrm{a}$ & $294.7 \pm 11.6 \mathrm{a}$ & $87.4 \pm 0.1 \mathrm{~b}$ & $93.1 \pm 7.2 \mathrm{~b}$ & $39.8 \pm 0.7 \mathrm{~b}$ & $42.4 \pm 0.7 \mathrm{~b}$ \\
\hline Ethyl hexanoate & $395.7 \pm 21.7 \mathrm{a}$ & $402.1 \pm 20.1 \mathrm{a}$ & $378.9 \pm 12.10 \mathrm{a}$ & $366.3 \pm 15.6 \mathrm{a}$ & $147.0 \pm 0.8 \mathrm{bc}$ & $154.5 \pm 14.4 \mathrm{~b}$ & $95.2 \pm 3.4 \mathrm{c}$ & $99.4 \pm 3.0 \mathrm{c}$ \\
\hline Ethyl 3-hexenoate & $2.4 \pm 0.2 \mathrm{~d}$ & $3.0 \pm 0.4 \mathrm{~d}$ & $1.6 \pm 1.1 \mathrm{~d}$ & $2.6 \pm 0.0 \mathrm{~d}$ & $8.5 \pm 0.0 \mathrm{ab}$ & $9.1 \pm 0.3 \mathrm{a}$ & $6.8 \pm 0.3 c$ & $7.0 \pm 0.2 \mathrm{bc}$ \\
\hline Ethyl enanthate & $3.1 \pm 0.1 \mathrm{a}$ & $3.1 \pm 0.1 \mathrm{a}$ & $3.0 \pm 0.0 \mathrm{a}$ & $2.8 \pm 0.1 \mathrm{a}$ & $1.6 \pm 0.0 \mathrm{~b}$ & $1.6 \pm 0.1 \mathrm{~b}$ & $1.5 \pm 0.1 \mathrm{~b}$ & $1.5 \pm 0.1 \mathrm{~b}$ \\
\hline Ethyl lactate & $3460.5 \pm 645.5 \mathrm{a}$ & $3190.9 \pm 907.9 \mathrm{a}$ & $3265.6 \pm 604.4 \mathrm{a}$ & $3387.4 \pm 131.4 \mathrm{a}$ & $4251.8 \pm 507.7 \mathrm{a}$ & $4276.0 \pm 520.7 \mathrm{a}$ & $4818.9 \pm 994.4 \mathrm{a}$ & $4173.8 \pm 365.8 \mathrm{a}$ \\
\hline Ethyl octanoate & $931.8 \pm 18.3 \mathrm{ab}$ & $955.1 \pm 6.3 \mathrm{a}$ & $858.0 \pm 39.6 \mathrm{~b}$ & $898.5 \pm 43.4 \mathrm{ab}$ & $506.8 \pm 18.8 \mathrm{c}$ & $527.3 \pm 2.3 \mathrm{c}$ & $370.5 \pm 1.0 \mathrm{~d}$ & $403.9 \pm 4.5 \mathrm{~d}$ \\
\hline Ethyl mesitylacetate & $1.1 \pm 0.1 \mathrm{a}$ & $1.0 \pm 0.0 \mathrm{a}$ & $1.0 \pm 0.0 \mathrm{a}$ & $1.0 \pm 0.0 \mathrm{a}$ & $0.5 \pm 0.0 \mathrm{~b}$ & $0.5 \pm 0.1 \mathrm{~b}$ & $0.4 \pm 0.0 \mathrm{~b}$ & $0.4 \pm 0.0 \mathrm{~b}$ \\
\hline Ethyl nonanoate & $4.0 \pm 0.2 \mathrm{a}$ & $4.3 \pm 0.1 \mathrm{a}$ & $4.2 \pm 0.7 \mathrm{a}$ & $3.7 \pm 1.1 \mathrm{a}$ & $7.5 \pm 0.6 \mathrm{a}$ & $7.9 \pm 2.4 \mathrm{a}$ & $7.0 \pm 0.6 \mathrm{a}$ & $6.1 \pm 1.5 \mathrm{a}$ \\
\hline E2H4MP & $16.0 \pm 0.59 c$ & $16.1 \pm 0.8 \mathrm{c}$ & $18.3 \pm 2.1 \mathrm{bc}$ & $18.1 \pm 0.2 \mathrm{bc}$ & $27.4 \pm 2.1 \mathrm{a}$ & $25.0 \pm 2.3 \mathrm{a}$ & $24.6 \pm 1.3 \mathrm{a}$ & $24.0 \pm 1.3 \mathrm{ab}$ \\
\hline Ethyl caprate & $738.6 \pm 0.1 \mathrm{~b}$ & $746.7 \pm 14.1 \mathrm{~b}$ & $888.1 \pm 54.1 \mathrm{a}$ & $922.8 \pm 46.2 \mathrm{a}$ & $620.0 \pm 58.9 \mathrm{bc}$ & $601.1 \pm 4.4 \mathrm{c}$ & $426.7 \pm 4.2 \mathrm{~d}$ & $462.0 \pm 1.6 \mathrm{~d}$ \\
\hline Ethyl benzoate & $1.7 \pm 0.1 \mathrm{a}$ & $1.8 \pm 0.1 \mathrm{a}$ & $1.7 \pm 0.1 \mathrm{a}$ & $1.8 \pm 0.1 \mathrm{a}$ & $1.7 \pm 0.2 \mathrm{a}$ & $1.7 \pm 0.1 \mathrm{a}$ & $1.5 \pm 0.1 \mathrm{a}$ & $1.6 \pm 0.0 \mathrm{a}$ \\
\hline Ethyl 9-decenoate & $17.1 \pm 1.1 \mathrm{a}$ & $18.4 \pm 1.7 \mathrm{a}$ & $16.2 \pm 2.3 \mathrm{a}$ & $18.9 \pm 1.3 \mathrm{a}$ & $3.9 \pm 0.0 \mathrm{~b}$ & $3.5 \pm 0.0 \mathrm{~b}$ & $3.4 \pm 0.1 \mathrm{~b}$ & $3.5 \pm 0.0 \mathrm{~b}$ \\
\hline Ethyl laurate & $135.3 \pm 2.6 \mathrm{bcd}$ & $137.3 \pm 4.6 \mathrm{abcd}$ & $173.2 \pm 13.4 \mathrm{bc}$ & $182.6 \pm 11.6 \mathrm{a}$ & $153.5 \pm 26.7 \mathrm{abc}$ & $119.4 \pm 2.7 \mathrm{~cd}$ & $96.8 \pm 0.0 \mathrm{~d}$ & $99.3 \pm 2.9 \mathrm{~d}$ \\
\hline Ethyl myristate & $24.0 \pm 0.5 \mathrm{a}$ & $23.3 \pm 5.7 \mathrm{a}$ & $22.9 \pm 4.2 \mathrm{a}$ & $27.5 \pm 1.1 \mathrm{a}$ & $22.3 \pm 3.9 \mathrm{a}$ & $20.8 \pm 1.6 \mathrm{a}$ & $19.4 \pm 2.1 \mathrm{a}$ & $24.8 \pm 3.9 \mathrm{a}$ \\
\hline Ethyl hexadecanoate & $38.7 \pm 1.0 \mathrm{bc}$ & $33.6 \pm 4.8 \mathrm{c}$ & $39.9 \pm 1.1 \mathrm{bc}$ & $40.0 \pm 2.2 \mathrm{bc}$ & $60.9 \pm 11.8 \mathrm{a}$ & $54.9 \pm 5.3 \mathrm{ab}$ & $61.4 \pm 3.3 \mathrm{a}$ & $70.4 \pm 1.0 \mathrm{a}$ \\
\hline Isobutyl acetate & $206.8 \pm 13.2 \mathrm{a}$ & $216.3 \pm 17.0 \mathrm{a}$ & $158.9 \pm 14.6 \mathrm{~b}$ & $154.6 \pm 0.4 \mathrm{~b}$ & $61.8 \pm 8.4 \mathrm{c}$ & $68.0 \pm 12.2 \mathrm{c}$ & $17.2 \pm 2.2 \mathrm{~d}$ & $33.4 \pm 6.7 \mathrm{~cd}$ \\
\hline Isopentyl acetate & $4878.4 \pm 338.0 \mathrm{a}$ & $5027.3 \pm 383.3 \mathrm{a}$ & $3790.5 \pm 325.2 \mathrm{~b}$ & $3603.7 \pm 160.7 \mathrm{~b}$ & $2382.1 \pm 5.8 \mathrm{c}$ & $2515.1 \pm 158.2 \mathrm{c}$ & $1110.7 \pm 11.2 \mathrm{~d}$ & $1182.4 \pm 48.9 \mathrm{~d}$ \\
\hline $\begin{array}{l}\text { Hexyl acetate } \\
\text { Hote }\end{array}$ & $9.10 \pm 0.5 \mathrm{a}$ & $9.3 \pm 0.3 \mathrm{a}$ & $6.8 \pm 0.4 \mathrm{~b}$ & $6.5 \pm 0.1 \mathrm{~b}$ & $1.3 \pm 0.1 \mathrm{~cd}$ & $2.3 \pm 0.0 \mathrm{c}$ & $1.2 \pm 0.7 \mathrm{~cd}$ & $0.7 \pm 0.5 \mathrm{~d}$ \\
\hline Heptyl acetate & $0.5 \pm 0.3 \mathrm{a}$ & $0.4 \pm 0.2 \mathrm{a}$ & $0.3 \pm 0.1 \mathrm{a}$ & $0.2 \pm 0.0 \mathrm{a}$ & $0.2 \pm 0.1 \mathrm{a}$ & $0.1 \pm 0.1 \mathrm{a}$ & $0.1 \pm 0.2 \mathrm{a}$ & $0.2 \pm 0.1 \mathrm{a}$ \\
\hline 2-Ethyl-1-hexanol acetate & $13.1 \pm 1.3 \mathrm{a}$ & $13.2 \pm 0.5 \mathrm{a}$ & $13.0 \pm 1.3 \mathrm{a}$ & $8.1 \pm 6.8 \mathrm{a}$ & $8.6 \pm 0.3 \mathrm{a}$ & $8.3 \pm 0.4 \mathrm{a}$ & $9.2 \pm 0.2 \mathrm{a}$ & $9.9 \pm 0.7 \mathrm{a}$ \\
\hline Octyl acetate & $7.5 \pm 0.5 \mathrm{ab}$ & $7.8 \pm 0.4 \mathrm{ab}$ & $7.2 \pm 0.9 \mathrm{ab}$ & $8.3 \pm 0.9 \mathrm{a}$ & $4.5 \pm 1.2 \mathrm{~b}$ & $5.7 \pm 0.3 \mathrm{ab}$ & $5.4 \pm 1.6 \mathrm{ab}$ & $5.3 \pm 0.6 \mathrm{ab}$ \\
\hline
\end{tabular}


Table 3. Cont.

\begin{tabular}{|c|c|c|c|c|c|c|c|c|}
\hline Volatile ( $\mu \mathrm{g} / \mathrm{L})$ & DF & DF-G & DP & DP-G & FF & FF-G & FP & FP-G \\
\hline Trimethylene acetate & $129.3 \pm 33.4 \mathrm{a}$ & $135.0 \pm 39.4 \mathrm{a}$ & $122.4 \pm 33.8 \mathrm{a}$ & $105.1 \pm 14.0 \mathrm{a}$ & $435.3 \pm 324.9 \mathrm{a}$ & $243.1 \pm 123.2 \mathrm{a}$ & $265.8 \pm 116.0 \mathrm{a}$ & $381.8 \pm 9.4 \mathrm{a}$ \\
\hline Isobutyl hexanoate & $0.6 \pm 0.2 \mathrm{ab}$ & $0.5 \pm 0.0 \mathrm{a}$ & $0.4 \pm 0.0 \mathrm{c}$ & $0.4 \pm 0.0 \mathrm{bc}$ & $\mathrm{ND}$ & $\mathrm{ND}$ & $\mathrm{ND}$ & ND \\
\hline Methyl octanoate & $5.2 \pm 0.1 \mathrm{a}$ & $5.5 \pm 0.1 \mathrm{a}$ & $5.1 \pm 0.2 \mathrm{a}$ & $3.4 \pm 2.4 \mathrm{a}$ & $2.6 \pm 0.1 \mathrm{a}$ & $2.7 \pm 0.0 \mathrm{a}$ & $2.3 \pm 0.0 \mathrm{a}$ & $2.4 \pm 0.0 \mathrm{a}$ \\
\hline Isopentyl hexanoate & $7.0 \pm 0.1 \mathrm{a}$ & $7.4 \pm 0.3 \mathrm{a}$ & $6.4 \pm 0.6 \mathrm{a}$ & $7.0 \pm 0.4 \mathrm{a}$ & $2.3 \pm 0.0 \mathrm{~b}$ & $2.3 \pm 0.1 \mathrm{~b}$ & $1.5 \pm 0.0 \mathrm{~b}$ & $1.7 \pm 0.0 \mathrm{~b}$ \\
\hline Isobutyl octanoate & $2.7 \pm 0.5 \mathrm{a}$ & $2.6 \pm 0.6 \mathrm{a}$ & $2.3 \pm 0.4 \mathrm{a}$ & $2.4 \pm 0.4 \mathrm{a}$ & $3.0 \pm 0.4 \mathrm{a}$ & $1.6 \pm 0.1 \mathrm{a}$ & $2.8 \pm 1.9 \mathrm{a}$ & $4.3 \pm 0.1 \mathrm{a}$ \\
\hline Methyl caprate & $12.2 \pm 0.2 \mathrm{a}$ & $12.1 \pm 0.1 \mathrm{a}$ & $18.3 \pm 1.4 \mathrm{a}$ & $18.9 \pm 1.2 \mathrm{a}$ & $6.8 \pm 1.1 \mathrm{a}$ & $6.7 \pm 0.1 \mathrm{a}$ & $3.4 \pm 0.1 \mathrm{a}$ & $4.5 \pm 0.3 \mathrm{a}$ \\
\hline Isoamyl octanoate & $9.4 \pm 0.3 \mathrm{ab}$ & $9.7 \pm 0.4 \mathrm{a}$ & $7.7 \pm 0.7 \mathrm{~b}$ & $8.3 \pm 0.8 \mathrm{ab}$ & $4.5 \pm 0.2 \mathrm{c}$ & $4.6 \pm 0.0 \mathrm{c}$ & $3.7 \pm 0.1 \mathrm{c}$ & $4.2 \pm 0.1 \mathrm{c}$ \\
\hline Diethyl succinate & $125.6 \pm 2.5 \mathrm{~b}$ & $126.4 \pm 13.2 \mathrm{~b}$ & $216.0 \pm 45.4 \mathrm{a}$ & $227.6 \pm 5.7 \mathrm{a}$ & $100.5 \pm 30.0$ & $87.5 \pm 13.5 \mathrm{~b}$ & $108.4 \pm 3.5 \mathrm{~b}$ & $92.0 \pm 9.5 \mathrm{~b}$ \\
\hline Isoamyl decanoate & $4.6 \pm 0.1 \mathrm{a}$ & $4.6 \pm 0.4 \mathrm{a}$ & $5.2 \pm 0.0 \mathrm{a}$ & $5.5 \pm 0.4 \mathrm{a}$ & $3.2 \pm 0.3 \mathrm{~b}$ & $2.9 \pm 0.0 \mathrm{bc}$ & $2.0 \pm 0.0 \mathrm{~d}$ & $2.2 \pm 0.0 \mathrm{~cd}$ \\
\hline IMDMMPo & $19.7 \pm 1.5 \mathrm{a}$ & $19.4 \pm 1.3 \mathrm{a}$ & $12.5 \pm 1.0 \mathrm{~b}$ & $13.3 \pm 0.4 \mathrm{~b}$ & $3.1 \pm 0.7 \mathrm{c}$ & $2.5 \pm 0.9 \mathrm{c}$ & $2.0 \pm 0.3 \mathrm{c}$ & $2.3 \pm 0.8 \mathrm{c}$ \\
\hline NA3MBE & $5.2 \pm 0.1 \mathrm{a}$ & $5.0 \pm 0.2 \mathrm{a}$ & $5.0 \pm 0.4 \mathrm{a}$ & $5.3 \pm 0.2 \mathrm{a}$ & $2.1 \pm 0.2 \mathrm{~b}$ & $1.9 \pm 0.0 \mathrm{~b}$ & $1.9 \pm 0.0 \mathrm{~b}$ & $1.8 \pm 0.0 \mathrm{~b}$ \\
\hline Total & $86200.3 \pm 1208.5 a$ & $89527.3 \pm 1927.2 a$ & $86020.1 \pm 6080.8 a$ & $82887.4 \pm 822.9 a$ & $56786.7 \pm 2501.9 b$ & $58602.3 \pm 2531.9 b$ & $59713.0 \pm 716.2 b$ & $64394.2 \pm 1452.0 \mathrm{~b}$ \\
\hline \multicolumn{9}{|l|}{ Others } \\
\hline Styrene & $97.1 \pm 1.7 \mathrm{ab}$ & $97.8 \pm 2.1 \mathrm{ab}$ & $83.3 \pm 3.5 b$ & $86.5 \pm 2.7 b$ & $93.5 \pm 3.5 \mathrm{ab}$ & $105.7 \pm 7.5 \mathrm{a}$ & $65.5 \pm 4.2 \mathrm{c}$ & $67.3 \pm 1.9 c$ \\
\hline$\alpha$-Ionene & $7.8 \pm 0.2 \mathrm{a}$ & $6.9 \pm 0.2 \mathrm{a}$ & $6.4 \pm 2.2 \mathrm{a}$ & $7.2 \pm 0.7 \mathrm{a}$ & $2.6 \pm 0.4 \mathrm{a}$ & $2.2 \pm 0.1 \mathrm{a}$ & $3.8 \pm 0.2 \mathrm{a}$ & $3.8 \pm 0.3 \mathrm{a}$ \\
\hline ODETMq & $2.7 \pm 0.1 \mathrm{c}$ & $2.8 \pm 0.1 \mathrm{c}$ & $2.6 \pm 0.0 \mathrm{~cd}$ & $2.7 \pm 0.2 \mathrm{c}$ & $\begin{array}{l}2.0 \pm 0.2 \mathrm{a} \\
6.0 \pm 0.2 \mathrm{~b}\end{array}$ & $6.7 \pm 0.0 \mathrm{ab}$ & $6.6 \pm 0.1 \mathrm{~b}$ & $7.5 \pm 0.5 \mathrm{a}$ \\
\hline$\alpha$-Cedrene & $2.1 \pm 0.0 \mathrm{a}$ & $2.0 \pm 0.1 \mathrm{a}$ & $1.2 \pm 0.2 \mathrm{a}$ & $1.4 \pm 0.2 \mathrm{a}$ & $0.2 \pm 0.0 \mathrm{~b}$ & $0.1 \pm 0.0 \mathrm{~b}$ & $0.1 \pm 0.0 \mathrm{~b}$ & $0.1 \pm 0.0 \mathrm{~b}$ \\
\hline$\alpha$-Calacorene & $6.2 \pm 0.1 \mathrm{a}$ & $5.8 \pm 0.2 \mathrm{ab}$ & $5.2 \pm 1.4 \mathrm{ab}$ & $5.9 \pm 0.5 \mathrm{ab}$ & $4.4 \pm 0.6 \mathrm{ab}$ & $3.9 \pm 0.0 \mathrm{~b}$ & $4.0 \pm 0.0 \mathrm{ab}$ & $3.9 \pm 0.2 \mathrm{~b}$ \\
\hline$\beta$-Ionone & $7.4 \pm 0.8 \mathrm{a}$ & $7.3 \pm 0.8 \mathrm{a}$ & $6.5 \pm 0.4 \mathrm{a}$ & $6.8 \pm 0.2 \mathrm{a}$ & $1.7 \pm 0.3 c$ & $1.8 \pm 0.2 \mathrm{c}$ & $4.5 \pm 0.0 \mathrm{~b}$ & $4.1 \pm 0.0 \mathrm{~b}$ \\
\hline DMUDL & $0.4 \pm 0.1 \mathrm{ab}$ & $0.4 \pm 0.1 \mathrm{ab}$ & $0.5 \pm 0.0 \mathrm{a}$ & $0.5 \pm 0.0 \mathrm{a}$ & $0.2 \pm 0.1 \mathrm{c}$ & $0.2 \pm 0.0 \mathrm{c}$ & $0.3 \pm 0.0 \mathrm{bc}$ & $0.3 \pm 0.0 \mathrm{bc}$ \\
\hline Cedrol & $0.5 \pm 0.1 \mathrm{a}$ & $0.4 \pm 0.0 \mathrm{a}$ & $0.3 \pm 0.0 \mathrm{~b}$ & $0.3 \pm 0.0 \mathrm{~b}$ & $\mathrm{ND}$ & $\mathrm{ND}$ & ND & $\mathrm{ND}$ \\
\hline Total & $124.1 \pm 1.0 a$ & $123.5 \pm 0.8 a$ & $106.0 \pm 7.77 a$ & $111.2 \pm 4.5 a$ & $108.7 \pm 1.9 a$ & $120.6 \pm 7.2 a$ & $84.8 \pm 4.0 b$ & $86.9 \pm 2.8 b$ \\
\hline
\end{tabular}

DF and DP indicate dried goji berry fermented wine made of free-run and pressed juice, respectively. DF-G and DP-G represent dried goji berry fermented wine made of free-run and pressed juice after the glycosidase treatment, respectively. FF and FP are fresh goji berry fermented wine made of free-run and pressed juice, respectively. FF-G and FP-G stand for fresh goji berry fermented wine made of free-run and pressed juice after the glycosidase treatment, respectively. * Different letters in the same row indicate significant differences at $p \leq 0.05$. **,**: "ND" and "TR" represent "not detected" and "trace amount", respectively. Data were expressed as the mean \pm standard deviation. 
Table 4. T-test on concentration of volatile compounds in wine made of fresh and dried berry fruit and two-factor variance analysis on concentration of volatile compounds in goji berry fermented wine with pressing and glycosidase treatments.

\begin{tabular}{|c|c|c|c|c|c|c|c|}
\hline \multirow{3}{*}{ Volatile } & \multirow{3}{*}{$\begin{array}{c}\text { T-test between } \\
\text { DW and FW }\end{array}$} & \multicolumn{3}{|c|}{ Dried Goji Berry Fermented Wine } & \multicolumn{3}{|c|}{ Fresh Goji Berry Fermented Wine } \\
\hline & & \multicolumn{3}{|c|}{$p$ Value } & \multicolumn{3}{|c|}{$p$ Value } \\
\hline & & Pressing & Glycosidase & Interaction & Pressing & Glycosidase & Interaction \\
\hline \multicolumn{8}{|l|}{ Volatile acids } \\
\hline DMECA & $16.35 * * *$ & 0.639 & 0.316 & 0.584 & 0.758 & 0.386 & 0.427 \\
\hline Acetic acid & $0.33 * * *$ & 0.582 & 0.527 & 0.890 & 0.145 & 0.341 & 0.706 \\
\hline Butanoic acid & $3.70 * * *$ & 0.990 & 0.994 & 0.924 & 0.710 & 0.624 & 0.651 \\
\hline$\alpha$-Methylbutyric acid & $3.47 * * *$ & 0.599 & 0.537 & 0.66 & 0.567 & 0.367 & 0.489 \\
\hline Octanoic acid & $1.51 * *$ & 0.626 & 0.225 & 0.784 & 0.559 & 0.506 & 0.732 \\
\hline Decanoic acid & 0.98 & 0.043 * & 0.100 & 0.223 & $0.047^{*}$ & 0.663 & 0.505 \\
\hline Total & $1.37 *$ & 0.052 & 0.097 & 0.313 & 0.107 & 0.546 & 0.687 \\
\hline \multicolumn{8}{|l|}{ Higher alcohols } \\
\hline 2-Methyl-1-propanol & $1.63 * * *$ & 0.648 & 0.498 & 0.789 & $0.001 * *$ & 0.934 & 0.829 \\
\hline Isopentyl alcohol & 0.96 & 1.000 & 0.273 & 0.720 & $0.0001 * * *$ & 0.619 & 0.765 \\
\hline Pentyl alcohol & $0.59 * * *$ & 0.278 & 0.598 & 0.164 & $0.014 *$ & 0.565 & 0.350 \\
\hline 1-Hexanol & $0.71 *$ & 0.362 & 0.487 & 0.235 & $0.000 * * *$ & $0.007 * *$ & 0.673 \\
\hline 2-Heptanol & $0.81 * * *$ & $0.014 *$ & 0.707 & 0.608 & 0.314 & 0.317 & 0.029 * \\
\hline 1-Octen-3-ol & $0.58 * *$ & 0.478 & $0.042 *$ & 0.777 & 0.557 & 0.617 & 0.475 \\
\hline 2-Ethyl-1-hexanol & $0.30 * *$ & $0.002 * *$ & 0.949 & 0.146 & $0.000 * * *$ & 0.633 & 0.261 \\
\hline$\left[R-\left(R^{*}, R^{*}\right)\right]-2,3-$ Butanediol & $0.24 * *$ & 0.655 & 0.746 & 0.821 & $0.001 * * *$ & 0.801 & 0.423 \\
\hline 1-Octanol & 0.46 ** & 0.458 & 0.754 & 0.832 & $0.000 * * *$ & 0.408 & 0.125 \\
\hline$\left[S-\left(R^{*}, R^{*}\right)\right]-2,3-$ Butanediol & $0.43 * *$ & 0.638 & 0.624 & 0.498 & 0.827 & 0.929 & 0.290 \\
\hline trans-2-Octenol & $0.56^{* * *}$ & 0.543 & 0.240 & 0.481 & 0.085 & 0.879 & 0.227 \\
\hline 1-Nonanol & $0.43^{* * *}$ & $0.010 * *$ & $0.000 * * *$ & $0.040 *$ & $0.005 * *$ & 0.300 & 0.278 \\
\hline Methionol & $1.73^{* * *}$ & 0.855 & 0.724 & 0.711 & 0.601 & 0.587 & 0.713 \\
\hline$(E)-5$-Decen-1-ol & $0.00 * *$ & 0 & 0 & 0 & $0.000 * * *$ & 0.077 & 0.384 \\
\hline Phenylethyl Alcohol & $1.49 * *$ & 0.874 & 0.939 & 0.872 & 0.972 & 0.52 & 0.718 \\
\hline Total & $0.36^{* *}$ & 0.659 & 0.704 & 0.750 & 0.877 & 0.833 & 0.405 \\
\hline
\end{tabular}


Table 4. Cont

\begin{tabular}{|c|c|c|c|c|c|c|c|}
\hline \multirow{3}{*}{ Volatile } & \multirow{3}{*}{$\begin{array}{l}\text { T-test between } \\
\text { DW and FW }\end{array}$} & \multicolumn{3}{|c|}{ Dried Goji Berry Fermented Wine } & \multicolumn{3}{|c|}{ Fresh Goji Berry Fermented Wine } \\
\hline & & \multicolumn{3}{|c|}{$p$ Value } & \multicolumn{3}{|c|}{$p$ Value } \\
\hline & & Pressing & Glycosidase & Interaction & Pressing & Glycosidase & Interaction \\
\hline \multicolumn{8}{|l|}{ Aldehyde/Ketones } \\
\hline Furfural & $39.11 * * *$ & 0.103 & 0.59 & 0.369 & 0.733 & 0.782 & 0.801 \\
\hline Decanal & 1.26 & 0.176 & $0.029 * *$ & 0.453 & 0.859 & 0.795 & 0.070 \\
\hline Safranal & $3.85 * * *$ & 0.111 & 0.728 & 0.810 & $0.000 * * *$ & 0.176 & 0.270 \\
\hline 2-Butyl-2-octenal & $1.25 * * *$ & 0.649 & 0.761 & 0.963 & 0.811 & 0.598 & 0.639 \\
\hline Isobutyl ketone & $16.21 * *$ & 0.682 & 0.088 & 0.126 & $0.003 * *$ & $0.003 * *$ & 0.378 \\
\hline TMCHN & $0.00 *$ & 0 & 0 & 0 & $0.002 * *$ & 0.463 & 0.463 \\
\hline $6 \mathrm{M} 5 \mathrm{H} 2 \mathrm{NE}$ & $* * *$ & 0.102 & 0.821 & 0.745 & 0 & 0 & 0 \\
\hline 2-Undecanone & $2.86 * * *$ & $0.001 * * *$ & 0.774 & 0.598 & $0.002 * *$ & 0.281 & 0.222 \\
\hline$\beta$-Cyclocitral & $1.46^{* * *}$ & $0.003 * *$ & 0.977 & 0.855 & $0.000 * * *$ & 0.836 & 0.055 \\
\hline Dihydropseudoionone & $2.38 * * *$ & 0.911 & 0.342 & 0.308 & $0.000 * * *$ & 0.260 & 0.167 \\
\hline 2-Acetylpyrrole & $* * *$ & 0.822 & 0.878 & 0.956 & 0 & 0 & 0 \\
\hline TMHAAL & $5.22 * * *$ & 0.916 & 0.426 & 0.619 & 0.851 & 0.396 & $0.043 *$ \\
\hline Total & $16.03 * * *$ & 0.065 & 0.191 & 0.182 & 0.879 & 0.812 & 0.259 \\
\hline \multicolumn{8}{|l|}{ Benzene } \\
\hline$\overline{\text { Isodurene }}$ & $3.86 * * *$ & 0.125 & 0.555 & 0.568 & $0.001 * *$ & 0.221 & 0.820 \\
\hline TEMLNE & $2.16 * * *$ & 0.858 & 0.839 & 0.238 & 0.052 & 0.158 & 0.896 \\
\hline DPPXL & $1.62 * * *$ & 0.258 & 0.711 & 0.352 & $0.044^{*}$ & 0.776 & 0.950 \\
\hline Naphthalene & $2.20 * * *$ & 0.193 & 0.063 & 0.154 & 0.137 & 0.416 & 0.439 \\
\hline TMDHPE & $2.78 * * *$ & 0.355 & 0.851 & 0.417 & $0.007^{* *}$ & 0.341 & 0.553 \\
\hline M2PB & $0.83^{* * *}$ & $0.019 * *$ & 0.842 & $0.024 * *$ & 0.629 & 0.522 & 0.420 \\
\hline Phenethyl acetate & $3.86^{* * *}$ & 0.055 & 0.857 & 0.577 & $0.008 * *$ & 0.228 & 0.461 \\
\hline$\beta$-Methylnaphthalene & $3.25 * * *$ & 0.882 & 0.903 & 0.269 & $0.029 *$ & 0.583 & 0.619 \\
\hline Benzyl alcohol & 0.75 & 0.884 & 0.989 & 0.840 & 0.027 * & 0.778 & 0.926 \\
\hline Ethyl dihydrocinnamate & $0.41 * * *$ & 0.677 & 0.600 & 0.821 & 0.477 & 0.223 & 0.498 \\
\hline
\end{tabular}


Table 4. Cont.

\begin{tabular}{|c|c|c|c|c|c|c|c|}
\hline \multirow{3}{*}{ Volatile } & \multirow{3}{*}{$\begin{array}{l}\text { T-test between } \\
\text { DW and FW }\end{array}$} & \multicolumn{3}{|c|}{ Dried Goji Berry Fermented Wine } & \multicolumn{3}{|c|}{ Fresh Goji Berry Fermented Wine } \\
\hline & & \multicolumn{3}{|c|}{$p$ Value } & \multicolumn{3}{|c|}{$p$ Value } \\
\hline & & Pressing & Glycosidase & Interaction & Pressing & Glycosidase & Interaction \\
\hline$\alpha$-Methylnaphthalene & $5.12 * * *$ & 0.753 & 0.858 & 0.376 & 0.097 & 0.179 & 0.286 \\
\hline 4-Tolylcarbinol & $1.59 * *$ & 0.650 & 0.549 & 0.884 & 0.441 & 0.580 & 0.504 \\
\hline $3 \mathrm{P} 4 \mathrm{HP}$ & $* * *$ & $0.001 * *$ & 0.179 & 0.498 & 0 & 0 & 0 \\
\hline Coumaran & $0.23 * * *$ & 0.342 & 0.651 & 0.793 & 0.677 & 0.424 & 0.402 \\
\hline 4-Ethylphenol & $0.16 * * *$ & 0.996 & 0.745 & $0.044 *$ & 0.072 & 0.278 & 0.892 \\
\hline 4-Vinylguaiacol & $0.53 * * *$ & 0.249 & 0.816 & 0.828 & 0.845 & 0.427 & 0.558 \\
\hline Total & $0.25 * * *$ & 0.738 & 0.900 & 0.980 & 0.117 & 0.312 & 0.787 \\
\hline \multicolumn{8}{|l|}{ Esters } \\
\hline Ethyl Acetate & $1.45 * * *$ & 0.418 & 0.961 & 0.267 & $0.016^{*}$ & 0.077 & 0.341 \\
\hline Ethyl butanoate & $5.41 * * *$ & 0.438 & 0.935 & 0.445 & 0.167 & 0.290 & 0.346 \\
\hline Ethyl hexanoate & $3.11 * * *$ & 0.104 & 0.818 & 0.494 & $0.001 * * *$ & 0.335 & 0.768 \\
\hline Ethyl 3-hexenoate & $0.31 * * *$ & 0.196 & 0.133 & 0.654 & $0.000 * *$ & 0.059 & 0.330 \\
\hline Ethyl enanthate & $1.94 * * *$ & 0.058 & 0.156 & 0.148 & $0.013 * *$ & 0.772 & 0.402 \\
\hline Ethyl lactate & $0.76 * *$ & 0.999 & 0.878 & 0.686 & 0.636 & 0.532 & 0.502 \\
\hline Ethyl octanoate & $2.01 * * *$ & $0.041 *$ & 0.218 & 0.714 & $0.000 * * *$ & $0.017 * *$ & 0.404 \\
\hline Ethyl mesitylacetate & $2.42 * * *$ & 0.229 & 0.444 & $0.038^{*}$ & 0.089 & 0.773 & 0.788 \\
\hline Ethyl nonanoate & $0.56 * * *$ & 0.682 & 0.810 & 0.441 & 0.337 & 0.800 & 0.576 \\
\hline E2H4MP & $0.68^{* * *}$ & 0.057 & 0.980 & 0.832 & 0.209 & 0.299 & 0.516 \\
\hline Ethyl caprate & $1.56 * * *$ & $0.003 * *$ & 0.451 & 0.632 & $0.001 * *$ & 0.717 & 0.266 \\
\hline Ethyl benzoate & $1.08 *$ & 0.432 & 0.183 & 0.859 & $0.037 *$ & 0.509 & 0.491 \\
\hline Ethyl 9-decenoate & $4.92 * * *$ & 0.907 & 0.170 & 0.586 & $0.009 * *$ & 0.024 * & $0.004 * *$ \\
\hline Ethyl laurate & $1.34 * *$ & $0.003 * *$ & 0.433 & 0.605 & $0.016 *$ & 0.172 & 0.128 \\
\hline Ethyl hexadecanoate & $0.61 * * *$ & 0.123 & 0.266 & 0.247 & 0.165 & 0.764 & 0.190 \\
\hline Isobutyl acetate & $4.09 * * *$ & $0.004 * *$ & 0.790 & 0.497 & $0.002 * *$ & 0.126 & 0.439 \\
\hline Isopentyl acetate & $2.41 * * *$ & $0.005 * *$ & 0.936 & 0.491 & $0.000 * * *$ & 0.156 & 0.629 \\
\hline
\end{tabular}


Table 4. Cont.

\begin{tabular}{|c|c|c|c|c|c|c|c|}
\hline \multirow{3}{*}{ Volatile } & \multirow{3}{*}{$\begin{array}{c}\text { T-test between } \\
\text { DW and FW }\end{array}$} & \multicolumn{3}{|c|}{ Dried Goji Berry Fermented Wine } & \multicolumn{3}{|c|}{ Fresh Goji Berry Fermented Wine } \\
\hline & & \multicolumn{3}{|c|}{$p$ Value } & \multicolumn{3}{|c|}{$p$ Value } \\
\hline & & Pressing & Glycosidase & Interaction & Pressing & Glycosidase & Interaction \\
\hline Hexyl acetate & $5.82 * * *$ & $0.000 * * *$ & 0.829 & 0.342 & 0.054 & 0.466 & 0.058 \\
\hline Heptyl acetate & $2.31 *$ & 0.283 & 0.487 & 0.989 & 0.658 & 0.983 & 0.454 \\
\hline 2-Ethyl-1-hexanol acetate & $1.32 *$ & 0.351 & 0.379 & 0.370 & $0.023 * *$ & 0.596 & 0.155 \\
\hline Octyl acetate & $1.47 * * *$ & 0.831 & 0.236 & 0.540 & 0.739 & 0.534 & 0.416 \\
\hline Trimethylene acetate & $0.37 * *$ & 0.457 & 0.811 & 0.634 & 0.911 & 0.783 & 0.300 \\
\hline Isobutyl hexanoate & $7.32 * * *$ & $0.004 * *$ & 0.095 & 0.639 & $0.000 * * *$ & 0.476 & 0.476 \\
\hline Methyl octanoate & $1.94 * *$ & 0.256 & 0.439 & 0.327 & $0.000 * * *$ & $0.033 * *$ & 0.940 \\
\hline Isopentyl hexanoate & $3.60 * * *$ & 0.149 & 0.162 & 0.722 & $0.000 * * *$ & 0.085 & $0.028 * *$ \\
\hline Methyl caprate & 0.81 & $0.000 * * *$ & 0.106 & 0.253 & 0.688 & 0.144 & 0.346 \\
\hline Isoamyl octanoate & $2.06 * * *$ & $0.022 *$ & 0.348 & 0.765 & 0.003 & 0.022 & 0.076 \\
\hline Diethyl succinate & $1.79 * *$ & $0.005 * *$ & 0.729 & 0.764 & 0.635 & 0.292 & 0.892 \\
\hline Isoamyl decanoate & $1.95 * * *$ & $0.027 * *$ & 0.469 & 0.55 & $0.001 * *$ & 0.950 & 0.143 \\
\hline IMDMMP & $6.60 * * *$ & $0.001 * *$ & 0.798 & 0.499 & 0.287 & 0.868 & 0.406 \\
\hline NA3MBE & $2.67 * * *$ & 0.756 & 0.954 & 0.255 & 0.059 & 0.262 & 0.456 \\
\hline Total & $1.44^{* * *}$ & 0.222 & 0.965 & 0.234 & 0.034 & 0.086 & 0.387 \\
\hline \multicolumn{8}{|l|}{ Others } \\
\hline$\overline{\text { Styrene }}$ & 1.10 & $0.002 * *$ & 0.355 & 0.527 & $0.000 * * *$ & 0.105 & 0.198 \\
\hline$\alpha$-Ionene & $2.48 * * *$ & 0.783 & 0.169 & 0.701 & 0.078 & 0.212 & 0.988 \\
\hline ODETM & $0.40 * * *$ & 0.407 & 0.185 & 0.865 & $0.023 *$ & $0.012 *$ & 0.622 \\
\hline$\alpha$-Cedrene & $1.92 * * *$ & 0.772 & 0.277 & 0.772 & 0.446 & 0.333 & 0.142 \\
\hline$\alpha$-Calacorene & $1.42 * * *$ & 0.435 & 0.787 & 0.372 & 0.418 & 0.232 & 0.454 \\
\hline$\beta$-Ionone & $2.32 * * *$ & 0.158 & 0.788 & 0.666 & $0.000 * * *$ & 0.135 & 0.131 \\
\hline DMUDL & $1.85 * * *$ & $0.024 *$ & 0.915 & 0.928 & 0.152 & 0.442 & 0.702 \\
\hline Cedrol & $* * *$ & $0.002 * *$ & 0.808 & 0.654 & 0 & 0 & 0 \\
\hline Total & $1.18 *$ & $0.011 *$ & 0.414 & 0.465 & $0.001 * *$ & 0.123 & 0.288 \\
\hline
\end{tabular}

The result of T-test between DW and DF is calculated using the volatile concentration in wine made of dried and fresh goji berry and presented with the concentration ratio. $p$ value of pressing effect is calculated using the volatile concentration in wine made of free-run and pressed juice. $p$ value of glycosidase effect is calculated using the volatile concentration in wine with and without glycosidase treatment. Interaction represent the interactive effect made of pressing and glycosidase treatments on volatile concentration in wine. ${ }^{* * *}$, and ${ }^{* * *}$ indicate the significance effect at $p \leq 0.05,0.01$, and 0.001 , respectively. 


\subsubsection{Higher Alcohols}

The higher alcohol content was significantly so in the fresh goji berry fermented wine (Table 3). Particularly, $\left[R-\left(R^{*}, R^{*}\right)\right]$ - and $\left[S-\left(R^{*}, R^{*}\right)\right]$-2,3-butanediol, pentyl alcohol, 1-hexanol, 2-heptanol, 2-ethyl-1-hexanol, 1-octanol, 1-nonanol, 1-octen-3-ol, trans-2-octenol, and E-5-decen-1-ol showed a higher concentration in the fresh goji berry fermented wine, whereas the dried goji berry resulted in a wine with higher concentrations of 2-methyl-1-propanol and methionol. 2,3-Butanediol (including both the $\left[R-\left(R^{*}, R^{*}\right)\right]$ and $\left.\left[S-\left(R^{*}, R^{*}\right)\right]\right)$ isomers) has been considered an important fermentation by-product that could alter the overall aroma of wine [24]. The obvious content differences in these wines might induce the different aromatic features. No significant differences on the content of 1-butanol, isopentyl alcohol, (Z)-3-hexen-1-ol, or 3-ethoxy-1-propanol were observed between the fresh and the dried goji berry fermented wines.

\subsubsection{Aldehydes and Ketones}

Aldehydes and ketones are considered the important aromatic compounds in fruits, and the drying process has been reported to enhance their accumulation in dried fruits [22]. Furfural has been reported to be produced by the dehydration of sugar under acidic conditions, and its content increases as the drying period increases [25]. 2-Acetylpyrrole was synthesized from proline, hydroxyproline, and/or sugar under Strecker degradation conditions, and this volatile exhibits a burnt scent [26]. Decanal is derived from the oxidation of oleic acid in fruits during the drying process [27]. This volatile contributes soapy and green lemon odors to the overall aroma [28]. The dried goji berry fermented wine contained significantly higher concentrations of furfural, safranal, 2-butyl-2-octenal, isobutyl ketone, 6-methyl-5-heptene-2-one, 2-undecanone, $\beta$-cyclocitral, dihydropseudo-ionone, 2-acetylpyrrole, and (2,6,6-trimethyl-2-hydroxycyclohexylidene)acetic acid lactone (Tables 3 and 4). Particularly, 6-methyl-5-heptene-2-one exists in a trace amounts in the fresh goji berry fermented wine, whereas the dried goji berry fermented wine contained this compound with much higher concentration. 6-Methyl-5-heptene-2-one is a product of the hydrolysis of carotenoids in fruits. We speculate that the drying process might result in the degradation of carotenoids, which leads to a higher concentration of 6-methyl-5-heptene-2-one in the dried goji berry fruit fermented wine.

\subsubsection{Volatile Acids}

Volatile acids are produced by the metabolism of sugars, lipids, and amino acids during the wine fermentation process [29]. Although these fatty acids exist in wine in small amounts, they contribute significantly fatty, pungent, rancid, fruity, and cheesy odors to wines [30,31]. No difference in the acid composition was observed in the goji berry fermented wines produced from the different raw materials, however, their concentrations showed significant differences (Tables 3 and 4). The dried goji berry fermented wine contained higher concentrations of 1,2-dimethyl-cyclopent-2-ene-carboxylic acid, butanoic acid, $\alpha$-methylbutyric acid, and octanoic acid. Particularly, the concentration of 1,2-dimethylcyclopent-2-enecarboxylic acid in the dried goji berry fermented wine was 16 times higher than that in the fresh berry fermented wine. The higher level of butanoic acid in the dried berry fermented wine might result from its accumulation during the fruit postharvest application [32]. The fresh goji berry fermented wine showed three times higher concentration of acetic acid than the dried goji berry fermented wine. These wines had similar levels of decanoic acid.

\subsubsection{Esters}

Esters are mainly formed as secondary products of yeast metabolism during the fermentation process [29]. Their levels in wine are mainly determined by those of their precursors in fruits [33]. It has been accepted that the drying process alters the composition of ester precursors in fruits [22] and as a result, a significant difference on the ester levels between fresh and the dried goji berry fermented wines might be expected. Ethyl acetate was the dominant ester in both goji wines, with a higher 
concentration in the dried berry fermented wine (Table 4). Similarly, the dried berry fermented wine also contained higher levels of the majority of the ester compounds, including 11 ethyl esters, six acetate esters, four fatty acid esters, and two other esters. Only six esters (ethyl 3-hexenoate, ethyl lactate, ethyl nonanoate, ethyl 2-hydroxy-4-methylpentanoate, ethyl hexadecanoate, and trimethylene acetate) exhibited higher concentrations in the fresh goji berry fermented wine. Both of the wines contained similar levels of ethyl myristate, isobutyl octanoate, and methyl caprate.

\subsubsection{Benzenes and Volatile Phenols}

Although the levels of benzenes were not as high as those of alcohols and esters in the goji berry fermented wines, a significant difference on their concentration was also observed (Table 4). The fresh goji berry fermented wine contained higher concentrations of methyl 2-(pentyloxy) benzoate, ethyl dihydrocinnamate, coumaran, 4-ethylphenol and 4-vinylguaiacol. On average, the concentrations of 4-ethylphenol and 4-vinylguaiacol were $1474 \mu \mathrm{g} / \mathrm{L}$ and $346 \mu \mathrm{g} / \mathrm{L}$ in the dried berry fermented wine, which corresponds to only about $16 \%$ and $54 \%$ of that in the fresh berry fermented wine, respectively. Considering the differences of their amount between these wines, we speculated that the drying process decreased the level of their precursors in the goji berry fruits [31-34]. Besides, the drying process also reduced the population of microorganisms, such as Dekkera bruxellensis, on the surface of goji berry fruits. These microorganisms have also been reported to convert $p$-coumaric and ferulic acid into 4-ethylphenol and 4-vinylguaiacol during the fermentation process [34,35]. The dried goji berry fermented wine possessed higher concentrations of isodurene, 1,1,4,6-tetramethylindane, 2,5-diisopropyl-p-xylene, naphthalene, 2,5,8-trimethyl-1,2-dihydronaphthalene, phenethyl acetate, $\beta$-methylnaphthalene, $\alpha$-methylnaphthalene, and 4-tolylcarbinol although these volatiles were all detected at low levels. Both the fresh and the dried goji berry fermented wines contained similar concentrations of ethyl cinnamate, 2,4-di-tert-butylphenol, and ethyl salicylate.

\subsubsection{Terpenes and Norisoprenoids}

Only six terpenoids and two $\mathrm{C}_{13}$-norisoprenoids were detected, and their total concentration was lower than those of the other volatiles (Tables 3 and 4). However, it has been confirmed that these volatile compounds play significant roles in contributing to the floral aroma of wine due to their low odor threshold ( $\beta$-ionone for example has an odor threshold of $0.09 \mu \mathrm{g} / \mathrm{L}$ ) [29]. Styrene was the dominant volatile in this aroma group. However, no significant concentration differences were observed in these fermented wines. The dried goji berry fermented wine showed higher levels of $\alpha$-ionene, $\alpha$-cedrene, $\alpha$-calacorene, $\beta$-ionone, and 2,6-dimethyl-2,6-undecadien-10-ol (0.5 to 7.1 $\mu \mathrm{g} / \mathrm{L})$. As reported, $\beta$-ionone can be formed by $\beta$-carotene degradation during heat treatment [36]. Obviously, the drying process resulted in the higher content of $\beta$-ionone in the dried berry and dried berry fermented wine. The average concentration of $\beta$-ionone in the dried berry fermented wine was about $7 \mu \mathrm{g} / \mathrm{L}$, about two times higher than that in the fresh berry fermented wine. The fresh goji berry fermented wine contained a higher concentration of 2,6,10,10-tetramethyl-1-oxaspiro[4.5]dec-6-ene.

\subsection{Effect of Pressing and Glycosidase on Volatile Composition in Goji Berry Wine}

Pressing during wine fermentation process can increase the extraction of secondary metabolites present in fruits [14]. However, the high level of these secondary metabolites in juice can produce a green-herbaceous aroma in wine, reducing its overall pleasant flavor [10]. Bound volatiles in fruits cannot contribute to the overall aroma of wine due to their odorless properties. Glycosidase has the capacity of cleaving the sugar moiety from bound volatiles, resulting in the formation of volatile aglycones. These released volatiles are able to exhibit flavor notes, and thus can enhance the overall aroma of wine [9]. 


\subsubsection{Effect on Dried Goji Berry Fermented Wine}

The pressing treatment resulted in significant differences in the concentration of 27 volatile compounds in the dried goji berry fermented wines (Table 4). For example, the wine fermented from the dried goji berry free-run juice contained higher concentrations of $\beta$-cyclocitral, 3-phenyl-4-hydroxyacetophenone, isopentyl acetate, ethyl octanoate, isobutyl acetate, isobutyl hexanoate, isoamyl octanoate, 1-[2-(isobutyryloxy)-1-methylethyl]-2,2-dimethylpropyl 2-methylpropanoate, styrene, and cedrol. Meanwhile, the pressing treatment significantly enhanced the concentrations of decanoic acid, 2-heptanol, 2-ethyl-1-hexanol, 2-undecanone, ethyl caprate, ethyl laurate, hexyl acetate, methyl caprate, diethyl succinate, isoamyl decanoate, and 2,6-dimethyl-2,6-undecadien-10-ol in the dried goji berry fermented wine. Decanoic acid (fatty and rancid odor) [37], ethyl caprate (fruity note) [37], and isopentyl acetate (fruity note) [38] exhibited concentrations higher than their thresholds in the dried goji berry fermented wine with the pressing treatment. The glycosidase treatment did not show a significant effect on the alteration of the aromatic profile in the dried goji berry fermented wine. For example, the increased contents of 1-octen-3-ol, 1-nonanol, and decanal in the dried goji berry fermented wine after the glycosidase treatment were not above their thresholds. The interactive effect from pressing and glycosidase treatments was also limited.

\subsubsection{Effect on Fresh Goji Berry Fermented Wine}

The pressing treatment showed a more obvious effect on the aromatic composition alteration in the fresh goji berry wine fermentation. For instance, 44 volatile compounds exhibited significant changes on their concentration in the wine after the pressing treatment (Table 4). More importantly, the majority of these volatile compounds were the dominant aromatic compounds in the wine. For example, 2-methyl-1-propanol, ethyl acetate, isopentyl alcohol, and 2,3-butanediol showed their highest contents in the fresh goji berry fermented wine after pressing treatment. 1-Hexanol is considered a significant aroma enhancer, with a concentration of $20-100 \mathrm{mg} / \mathrm{L}$ in wine $[39,40]$. The pressing treatment significantly increased its concentration in the wine, which could contribute its positive odor features in the wine aroma. Glycosidase did not significantly enhance the aromatic levels in the fresh goji berry fermented wine. Six volatiles showed concentration differences in the wines with and without the glycosidase treatment. However, none of them exhibited an OAV value above 1 . No significant effect of the interaction between pressing and glycosidase treatments was observed either.

We speculate that the fresh goji berries, compared to the dried goji berries, possessed more intact cell structures, which limits the extraction of volatile compounds and their precursors in the fresh berries during the vinification process. However, the pressing treatment might destroy the cell structure to release more aromatic compounds and their precursors, increasing their levels in the wine. Additionally, we also speculate that bound volatiles might exist in low levels in goji berries, since the application of glycosidase did not exert a significant effect on the release of more algycones in the goji berry wines. It should be noted that the glycosidase AR2000 used in this study is a non-specific glycosidase that possesses $\alpha$-L-arabinofuranosidase, $\alpha$-L-arabinopyranosidase, $\alpha$-L-rhamnosidase, and $\alpha$-D-glucosidase activity [41].

\subsection{Principal Component Analysis (PCA)}

The differentiation of these wines regarding their aromatic profile was observed in the principal component analysis (PCA) with the first two principal components (PC1 and PC2, Figure 1). PC1 represented $68.6 \%$ of the total variance, whereas $8.5 \%$ of the variance was explained by PC2. PC1 significantly differentiated between the wines made from dried and fresh goji berries due to the negative relation with 20 esters, nine benzenes, eight aldehydes/ketones, six other volatiles, three acids, and two alcohols (volatiles with a loading value below -0.1). Meanwhile, this differentiation was also positively related with four esters, three benzenes, three alcohols, two acids, one aldehyde/ketone, 
and one other volatile (volatiles with a loading value above 0.1 ). In addition, a distinction between the fresh goji berry fermented wine made of the free-run and the pressed juice was observed in PC2 according to the relation with isopentyl alcohol (loading value, -0.3166$)$, styrene $(-0.2733)$, benzyl alcohol (0.26244), 1-octanol (0.24277), (E)-5-decen-1-ol (0.22878), 1-hexanol (0.2413), and $\beta$-cyclocitral (0.21834). The PCA analysis indicated that the goji raw material (fresh vs dried) exerted a primary role in differentiating the aromatic profile of these wines, whereas pressing treatment affected the volatiles profiles of these wines.
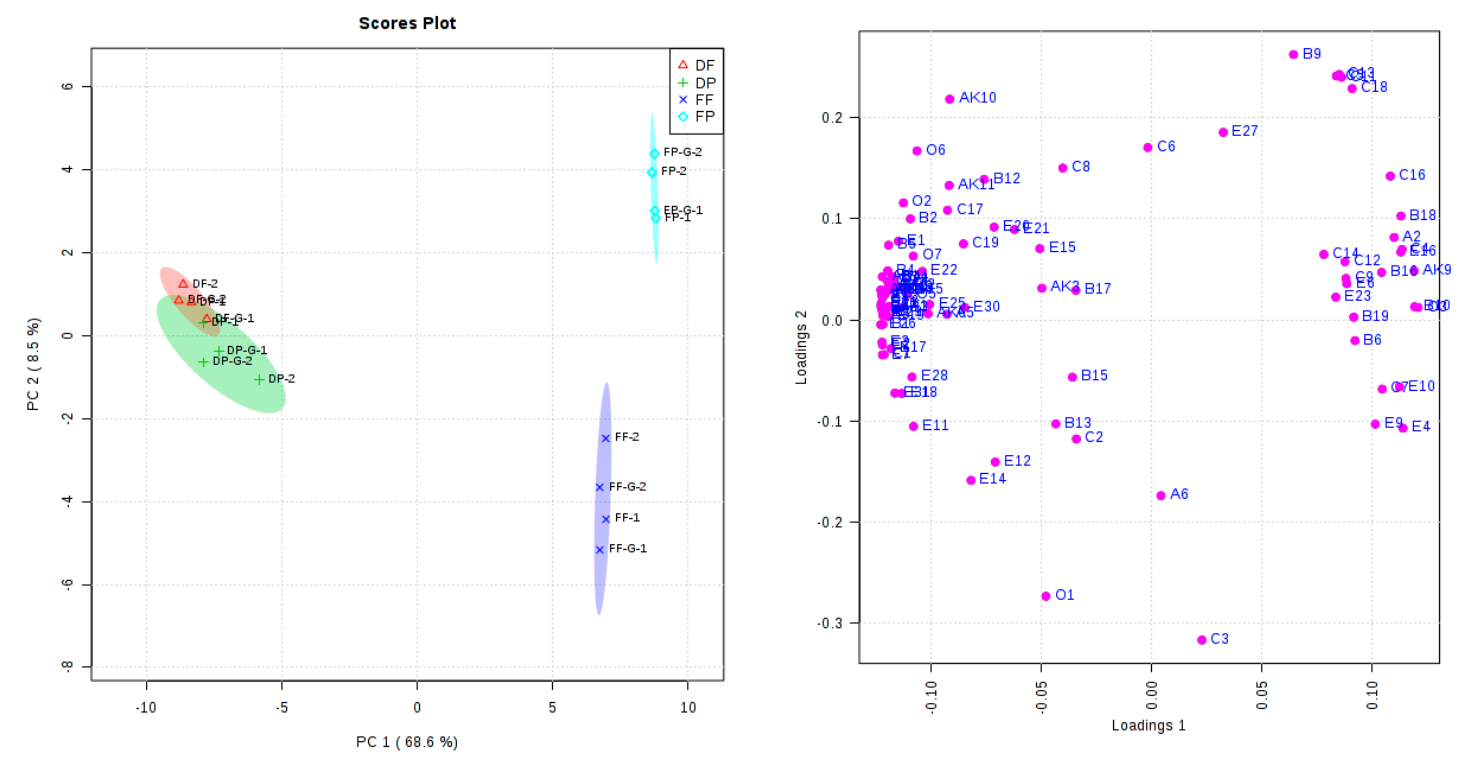

Figure 1. Principal component analysis (PCA) of goji berry fermented wine made of fresh and dried goji berry under pressing and glycosidase treatment. Left-Scores plot; Right-Loading plot; Volatiles with abbreviation of A1-O8 in loading plot are named in Table 3. "1", "2" in scores plot represent two replicates for each goji berry fruit wine fermentation treatment.

\subsection{Aroma Profile of Goji Berry Fermented Wine}

The aroma profile of the goji berry fermented wine was established by calculating the total OAV of the odorants, which was used to better understand its aromatic feature. The aromatic odorants were grouped into fruity, floral, herbaceous (or vegetal), caramel, fatty, and chemical aroma series. In the goji berry fermented wine, 17 volatile compounds showed concentrations higher than their odor threshold. These volatiles included six esters, five alcohols, three acids, one $\mathrm{C}_{13}$-norisoprenoid, and one volatile phenol (Table 5). Particularly, $\beta$-ionone showed the highest OAV value in the wine among these odorants, indicating that its violet note might be significantly incorporated into the goji berry fermented wine [32]. Isopentyl acetate reached the highest OAV value in the dried goji berry fermented wine, indicating that sweet and fruity notes could be enhanced in the wine [38]. The fresh goji berry fermented wine showed a strong horsy, leather, medicinal, smoky, barnyard, animal, and sweaty odor due to the high concentration of 4-ethylphenol [42-44]. In addition, there were 11 volatiles in the wine with its OAV value between 0.1 and 1 . These indicated that these volatiles might not directly contribute to the overall aroma of wine but just improve the wine aroma complexity [29]. 
Table 5. Odor activity value (OAVs) of volatile compound in goji berry fermented wines.

\begin{tabular}{|c|c|c|c|c|c|c|c|c|c|c|c|}
\hline Volatile & Threshold ( $\mu \mathrm{g} / \mathrm{L})$ & Aroma Descriptor & Aroma Series & DF & DF-G & DP & DP-G & FF & FF-G & FP & FP-G \\
\hline Butanoic acid & $173[45]$ & Butter, cheese, stinky [45] & $1[46]$ & $8.7 \mathrm{a}$ & $8.6 \mathrm{a}$ & $8.6 \mathrm{a}$ & $8.7 \mathrm{a}$ & $2.4 \mathrm{~b}$ & $2.4 \mathrm{~b}$ & $2.4 \mathrm{~b}$ & $2.2 \mathrm{~b}$ \\
\hline Octanoic acid & $500[37]$ & Rancid, cheese, fatty acid [28] & $1[28]$ & $2.0 \mathrm{a}$ & $1.6 \mathrm{a}$ & $2.1 \mathrm{a}$ & $1.8 \mathrm{a}$ & $1.5 \mathrm{a}$ & $1.2 \mathrm{a}$ & $1.2 \mathrm{a}$ & $1.1 \mathrm{a}$ \\
\hline Decanoic acid & 1000 [37] & Fatty, rancid [37] & $1[46]$ & $2.7 \mathrm{a}$ & $2.2 \mathrm{a}$ & $6.1 \mathrm{a}$ & $3.3 \mathrm{a}$ & $5.0 \mathrm{a}$ & $4.2 \mathrm{a}$ & $2.6 \mathrm{a}$ & $2.8 \mathrm{a}$ \\
\hline Isopentyl alcohol & $30000[47]$ & Solvent, sweety, alcohol, polish [38] & $1,2[38]$ & $6.3 \mathrm{~b}$ & $6.2 \mathrm{~b}$ & $6.3 \mathrm{~b}$ & $6.1 \mathrm{~b}$ & $7.2 \mathrm{a}$ & $7.2 \mathrm{a}$ & $5.8 \mathrm{~b}$ & $5.8 \mathrm{~b}$ \\
\hline$\left[R-\left(R^{*}, R^{*}\right)\right]-2,3$-Butanediol & $150000[28]$ & Fruit, sweet, butter [28] & $1,2,3[28]$ & $2.5 \mathrm{~b}$ & $2.4 \mathrm{~b}$ & $2.3 \mathrm{~b}$ & $1.9 \mathrm{~b}$ & $11.8 \mathrm{a}$ & $6.5 \mathrm{a}$ & $8.8 \mathrm{a}$ & $11.6 \mathrm{a}$ \\
\hline$\left[S-\left(R^{*}, R^{*}\right)\right]-2,3-$ Butanediol & $150000[28]$ & Fruit, sweet, butter [28] & $1,2,3[28]$ & $0.8 \mathrm{a}$ & $0.8 \mathrm{a}$ & $0.8 \mathrm{a}$ & $0.6 \mathrm{a}$ & $2.0 \mathrm{a}$ & $1.3 \mathrm{a}$ & $1.4 \mathrm{a}$ & $2.3 \mathrm{a}$ \\
\hline Methionol & $1000[28]$ & Cabbage, cooked potato, garlic [28] & $4[28]$ & $1.3 \mathrm{a}$ & $1.2 \mathrm{a}$ & $1.3 \mathrm{a}$ & $1.3 \mathrm{a}$ & $0.8 \mathrm{a}$ & $0.5 \mathrm{a}$ & $0.8 \mathrm{a}$ & $0.8 \mathrm{a}$ \\
\hline Phenylethyl Alcohol & $14000[28]$ & Roses, honey [28] & $5[28]$ & $1.5 \mathrm{a}$ & $1.4 \mathrm{a}$ & $1.4 \mathrm{a}$ & $1.4 \mathrm{a}$ & $1.1 \mathrm{a}$ & $0.8 \mathrm{a}$ & $1.0 \mathrm{a}$ & $0.9 \mathrm{a}$ \\
\hline Ethyl cinnamate & $1.1[47]$ & Flowery, balsamic [48] & $5[46]$ & $1.7 \mathrm{a}$ & $2.3 \mathrm{a}$ & $2.2 \mathrm{a}$ & $2.7 \mathrm{a}$ & $1.9 \mathrm{a}$ & $2.3 \mathrm{a}$ & $1.6 \mathrm{a}$ & $2.0 \mathrm{a}$ \\
\hline 4-Ethylphenol & $440[28]$ & Phenolic, leather [28] & $6[28]$ & $3.3 \mathrm{~b}$ & $3.4 \mathrm{~b}$ & $3.4 \mathrm{~b}$ & $3.3 \mathrm{~b}$ & $18.6 \mathrm{a}$ & $13.7 \mathrm{ab}$ & $26.5 \mathrm{a}$ & $22.7 \mathrm{a}$ \\
\hline Ethyl Acetate & $5000[8,49]$ & Spicy, pineapple, fruity [38] & $3[46]$ & $6.2 \mathrm{a}$ & $6.5 \mathrm{a}$ & $6.3 \mathrm{a}$ & $6.0 \mathrm{a}$ & $4.0 \mathrm{~b}$ & $4.1 \mathrm{~b}$ & $4.4 \mathrm{~b}$ & $4.8 \mathrm{~b}$ \\
\hline Ethyl hexanoate & $14[47]$ & Banana, green, apple [28] & $3[28]$ & $4.9 \mathrm{a}$ & $5.0 \mathrm{a}$ & $4.7 \mathrm{a}$ & $4.6 \mathrm{a}$ & $1.8 \mathrm{bc}$ & $1.9 \mathrm{~b}$ & $1.2 \mathrm{c}$ & $1.2 \mathrm{c}$ \\
\hline Ethyl octanoate & $5[28]$ & Sweet, floral, fruity, banana, pear [28] & $3,5[46]$ & $1.6 \mathrm{ab}$ & $1.6 \mathrm{a}$ & $1.5 \mathrm{~b}$ & $1.5 \mathrm{ab}$ & $0.9 \mathrm{c}$ & $0.9 \mathrm{c}$ & $0.6 \mathrm{~d}$ & $0.7 \mathrm{~d}$ \\
\hline Ethyl caprate & $200[37]$ & Brandy, fruity, grape [37] & $3[37]$ & $3.7 \mathrm{~b}$ & $3.7 \mathrm{~b}$ & $4.4 \mathrm{a}$ & $4.6 \mathrm{a}$ & $3.1 \mathrm{bc}$ & $3.0 \mathrm{c}$ & $2.1 \mathrm{~d}$ & $2.3 \mathrm{~d}$ \\
\hline Isopentyl acetate & 30 [47] & Sweety, fruity [38] & $2,3[46]$ & $30.5 \mathrm{a}$ & $31.4 \mathrm{a}$ & $23.7 \mathrm{~b}$ & $22.5 \mathrm{~b}$ & $14.9 \mathrm{c}$ & $15.7 \mathrm{c}$ & $6.9 \mathrm{~d}$ & $7.4 \mathrm{~d}$ \\
\hline$\beta$-Ionone & $0.09[47]$ & Violet [32] & $5[46]$ & $92.6 \mathrm{a}$ & $91.6 \mathrm{a}$ & $80.7 \mathrm{a}$ & 84.8 & $21.8 \mathrm{c}$ & $21.8 \mathrm{c}$ & $56.8 \mathrm{~b}$ & $50.6 \mathrm{~b}$ \\
\hline
\end{tabular}

DF and DP indicate dried goii berry fermented wine made of free-run and pressed juice, respectively. DF-G and DP-G represent dried goji berry fermented wine made of free-run and

pressed juice after the glycosidase treatment, respectively. FF and FP are fresh goji berry fermented wine made of free-run and pressed juice, respectively. FF-G and FP-G stand for fresh goji berry fermented wine made of free-run and pressed juice after the glycosidase treatment, respectively. Volatile compounds with available odor threshold and aroma descriptor from literature and OAV above 1 are listed. 1,2,3,4,5, and 6 in aroma series column represent fatty, caramel, fruity, herbaceous (or vegetal), floral, and chemical note, respectively. 
Regarding the specific aroma group (Figure 2), the goji berry fermented wine expressed floral aroma features, followed by fruity and caramel notes. These aroma attributes mainly resulted from the predominance of $\beta$-ionone and esters in the wine. 4-Ethylphenol and 4-vinylguaiacol featured chemical notes [44]. The fresh goji berry fermented wine, especially the wine obtained after the pressing treatment, exhibited a much stronger chemical odor due to the higher concentration of 4-ethylphenol and 4-vinylguaiacol. Moreover, the dried goji berry fermented wines showed significantly stronger floral odors, and high levels of fruity and caramel aromas. It was also worth noting that these features were much more obvious in the wines fermented from the free-run juice. These indicated that the better aromatic quality of goji berry wine was made from the free-run juice of the dried goji berry. These goji berry fermented wines exhibited little herbaceous flavor notes, and no significant differences were found among these wines.

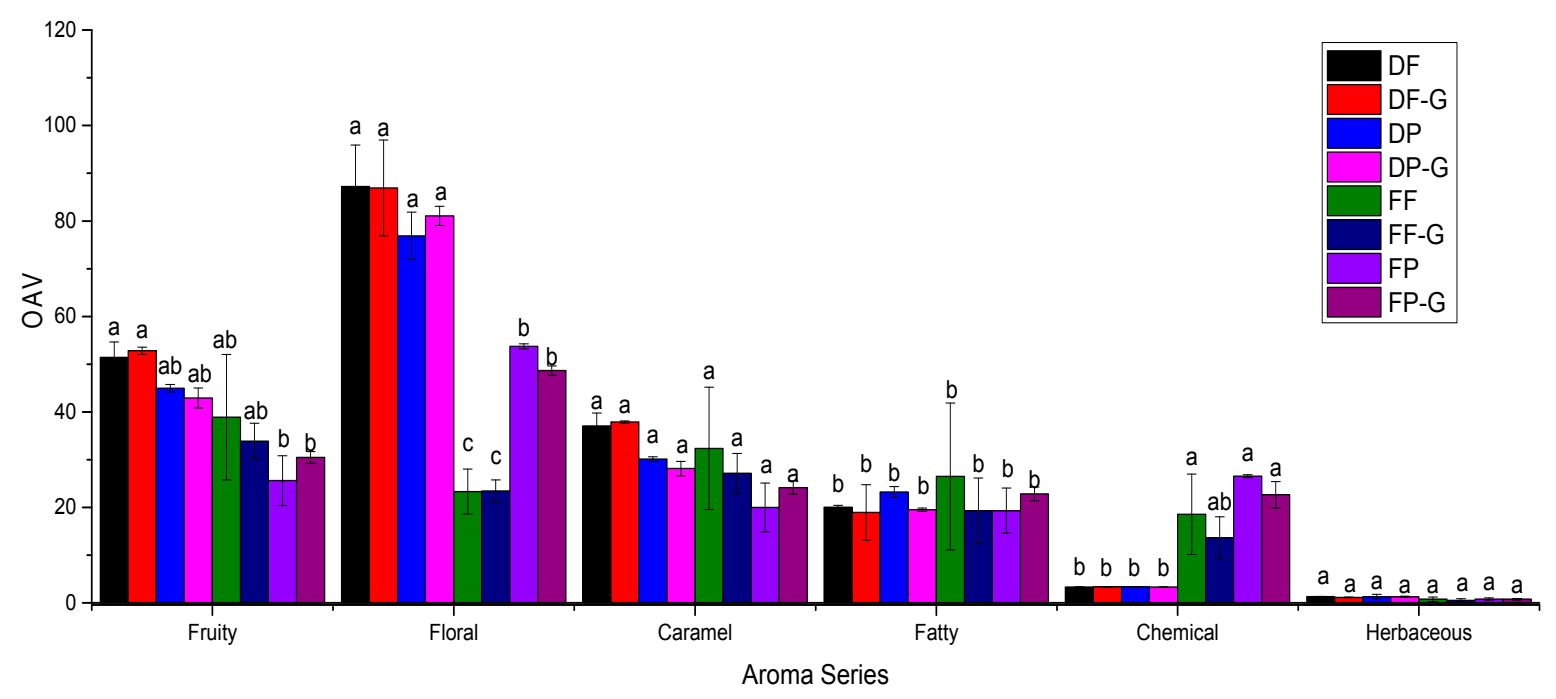

Figure 2. Total OAV value in each aroma group in goji berry fermented wine. The OAV value is calculated by summing up the OAV value of volatile in each aroma group. Different letters indicate significant difference of these wines in each aroma group at $p \leq 0.05$ of aroma series of goji wines.

\section{Materials and Methods}

\subsection{Samples and Reagents}

Fully ripe goji berries (Lycium barbarum L.) were harvested in the Ningxia growing region (Yinchuan City, $106.27^{\circ} \mathrm{E}, 28.47^{\circ} \mathrm{N}$ ) by Senmiao Corporation (Yinchuan, China) in the middle of July of 2015. The fresh goji berries were processed by Senmiao Corporation into dry goji berries through a hot-air drying process as well. Both fresh and the dry goji berries were used for the goji berry wine-making. A commercial Saccharomyces cerevisiae strain Red Fruit ${ }^{\circledR}$, pectinase, and potassium metabisulfite were purchased from Enartis (San Martino, Trecate, Italy). Glycosidase AR 2000 was a product from DSM Food Specialties (Delft, The Netherlands). Deionized water was purified by a Milli-Q purification system (Millipore, Bedford, MA, USA). Sodium hydroxide, diammonium phosphate, sodium chloride, ammonium sulfate, sodium sulfate, tartaric acid, ethanol, and sucrose were purchased from Beijing Chemical Works (Beijing, China). The standards of volatiles were purchased from Sigma-Aldrich (St. Louis, MO, USA). 


\subsection{Wine-Making Process}

\subsubsection{Wine Made of Dried Goji Berry}

Dried goji berries (40 kg, about $75 \%$ water loss) were mixed with water $(120 \mathrm{~L})$ in a $200 \mathrm{~L}$ stainless steel fermenter. Before the physicochemical parameter adjustment, the dried goji berry must had a sugar content of 180.5 glucose equivalent $\mathrm{g} / \mathrm{L}$, titratable acidity of $8.6 \mathrm{~g} / \mathrm{L}$, and a pH of 4.76 . Afterwards, $4 \mathrm{~g} / \mathrm{hL}$ pectinase and $45 \mathrm{mg} / \mathrm{L}$ potassium metabisulfite were added to the fermenter. The resultant mixture was maintained in the fermenter at $10^{\circ} \mathrm{C}$ for $24 \mathrm{~h}$, followed by mixing with diammonium phosphate $(0.21 \mathrm{~g} / \mathrm{L})$. After adjusting the sugar content to $230 \mathrm{~g} / \mathrm{L}$ using sucrose and $\mathrm{pH}$ to 3.8 using tartaric acid, the activated Red Fruit ${ }^{\circledR}$ S. cerevisiae $(20 \mathrm{~g} / \mathrm{hL})$ was added to initiate fermentation. The fermentation was maintained at $20^{\circ} \mathrm{C}$, and monitored by measuring the relative density of the must. When the relative density reached around 1.030, the goji berry juice was separated from the pomace as the free-run juice and transferred to another fermenter to continue the fermentation. The pomace was pressed three times under a $0.8 \mathrm{Mpa}$ for $5 \mathrm{~min}$ each time on a pneumatic presser (KSC125X400, Tungming Pneumatic Co., Ltd., Dongguan, China) to yield the pressed juice. Afterwards, the pressed juice was separated from the pomace and maintained in the fermenter for the fermentation. The fermentation of the free-run juice and the pressed juice was considered terminated when the density remained the same in three consecutive days to produce the dried goji berry free-run juice fermented wine (DF) and the dried goji berry pressed juice fermented wine (DP). These wines were then stored for 1 month at $2{ }^{\circ} \mathrm{C}$ in the fermenter filled with $\mathrm{CO}_{2}$ for better clarity, and then filtered through $0.45 \mu \mathrm{m}$ filters. Afterwards, the DF and the DP wine were treated with $4 \mathrm{~g} / \mathrm{hL}$ glycosidase AR 2000 at $15{ }^{\circ} \mathrm{C}$ in the darkness for one week to result in the glycosidase treated dried goji berry free-run juice fermented wine (DF-G) and the glycosidase treated dried goji berry pressed juice fermented wine (DP-G) according to our preliminary study. Each fermentation was conducted in duplicate, and a total of 8 goji berry wine samples were obtained, including DF (DF-1 and DF-2 as duplicates of DF), DP (DP-1 and DP-2), DF-G (DF-G-1 and DF-G-2), and DP-G (DP-G-1 and DP-G-2).

\subsubsection{Wine Made of Fresh Goji Berry}

The fresh goji berry fermentation was conducted using a similar approach as the dried goji berry wine fermentation. Before the adjustments, the fresh goji berry fruit juice had a sugar content of $193.5 \mathrm{~g}$ glucose equivalent/L, titratable acidity of $8.4 \mathrm{~g} / \mathrm{L}$, and a $\mathrm{pH}$ of 4.83 . Briefly, the fresh berries $(160 \mathrm{~kg})$ were crushed to yield the fresh goji berry must. The must was transferred into a $200 \mathrm{~L}$ stainless steel fermenter. Water $(10 \mathrm{~L})$ was added to the must to help mix it with the enzyme for a better fermentation performance. Afterwards, $4 \mathrm{~g} / \mathrm{hL}$ pectinase and $45 \mathrm{mg} / \mathrm{L}$ potassium metabisulfite was immediately added. The resultant mixture was maintained in the fermenter at $10{ }^{\circ} \mathrm{C}$ for $24 \mathrm{~h}$. Afterwards, $230 \mathrm{~g} / \mathrm{L}$ sucrose was adjusted and the $\mathrm{pH}$ was adjusted to $3.8 \mathrm{using}$ tartaric acid. The fermentation was initiated by adding activated Red Fruit ${ }^{\circledR}$ S. cerevisiae $(20 \mathrm{~g} / \mathrm{hL})$ to the must and maintained at $20^{\circ} \mathrm{C}$. The fresh goji berry free-run juice was collected when the relative density reached around 1.030. The free-run juice was transferred to another fermenter for the fermentation. The fresh goji berry pressed juice was collected using the same pressing procedure applied to the dried goji fruit fermentation, and then fermented in the same fermenter. The fresh goji berry free-run juice fermented wine (FF) and the fresh goji berry pressed juice fermented wine (FP) were yielded after their density remained the same in three consecutive days of the fermentation. These wines were then stored for 1 month at $2{ }^{\circ} \mathrm{C}$ in the fermenter filled with $\mathrm{CO}_{2}$ for better clarity, and then filtered through $0.45 \mu \mathrm{m}$ filters. Afterwards, the FF and the FP wine were treated with $4 \mathrm{~g} / \mathrm{hL}$ glycosidase AR 2000 at $15^{\circ} \mathrm{C}$ in the darkness for one week to yield the glycosidase treated fresh goji berry free-run juice fermented wine (FF-G) and the glycosidase treated fresh goji berry pressed juice fermented wine (FP-G), respectively. Each fermentation was conducted in duplicate, and a total of 8 goji berry wine samples were obtained, including FF (FF-1 and FF-2 as duplicates of DF), FP (FP-1 and FP-2), FF-G (FF-G-1 and FF-G-2), and FP-G (FP-G-1 and FP-G-2). 
The ethanol content of the samples were analyzed by a FOSS WineScan instrument (FOSS, Hillerød, Denmark). The physicochemical parameters of the goji wines, including titratable and volatile acidity, residual sugar content, total and free $\mathrm{SO}_{2}$ and $\mathrm{pH}$ were determined according to the National Standard of the People's Republic of China (GB/15038-2006, 2006). The polysaccharide content of the wines was analyzed according to the phenol-sulfuric acid method [50]. The physicochemical parameters of the wines were listed in Table 1.

\subsection{Volatile Compound Analyses}

A headspace solid-phase micro-extraction was used to extract volatile compounds from the wine according to a published method [51]. Briefly, the wine sample $(5.0 \mathrm{~mL})$ was mixed with $\mathrm{NaCl}(1 \mathrm{~g})$ and $1.0018 \mathrm{~g} / \mathrm{L}$ 4-methyl-2-pentanol (the internal standard, $10 \mu \mathrm{L}$ ) in a $15 \mathrm{~mL}$ vial containing a stirring bar. The vial was then capped with a PTFE-silicon septum and heated at $40{ }^{\circ} \mathrm{C}$ for 30 min under agitation. Afterwards, a SPME fiber (50/30 $\mu \mathrm{m}$ DVB/Carboxen/PDMS, Supelco, Bellefonte, PA, USA) was placed into the head-space of the mixture in the vial to extract the volatile compounds for $30 \mathrm{~min}$ under the same agitation and temperature. Finally, the fiber was removed from the head-space of the mixture and immediately inserted into the GC injector for volatile desorption for $8 \mathrm{~min}$. An Agilent 6890 GC interfaced with an Agilent 5975B MS (Agilent Technologies, Santa Clara, CA, USA) was used to analyze the volatile composition in the wine. The volatile compounds were separated on a HP-INNOWAX capillary column $(60 \mathrm{~m} \times 0.25 \mathrm{~mm}$ id, $0.25 \mu \mathrm{m}$ film thickness, J\&W Scientific, Folsom, CA, USA) under a carrier gas (helium) flow rate at $1 \mathrm{~mL} / \mathrm{min}$ with a splitless GC inlet mode. The oven temperature program was as follows: $50{ }^{\circ} \mathrm{C}$ held for $1 \mathrm{~min}$ and then rose to $220^{\circ} \mathrm{C}$ at $3{ }^{\circ} \mathrm{C} / \mathrm{min}$ and held at $220^{\circ} \mathrm{C}$ for $5 \mathrm{~min}$. A voltage of $70 \mathrm{eV}$ was set in the electron impact mode and the mass scan was conducted from $m / z$ 20-450 using a selective ion mode. The analysis for each wine sample was conducted in duplicate.

$\mathrm{A} \mathrm{C}_{7}-\mathrm{C}_{24} n$-alkane series (Supelco) and the same chromatographic procedure was used to calculate the retention indices. The identification of volatile compounds with the available standard was performed by matching their retention time and mass spectrum with their corresponding standard. For the volatile compounds without the available standard, these volatiles were tentatively identified according to their mass spectrum and retention indices with the standard NIST 11 library and NIST Standard Reference Database.

The reference standards were dissolved in a synthetic matrix solution containing $9 \mathrm{~g} / \mathrm{L}$ tartaric acid, $12 \%(v / v)$ ethanol, and $10 \mathrm{~g} / \mathrm{L}$ glucose. The matrix was adjusted to $\mathrm{pH} 3.8 \mathrm{using} 5 \mathrm{~mol} / \mathrm{L} \mathrm{NaOH}$, and then $10 \mu \mathrm{L}$ internal standard solution (4-methyl-2-pentanol, $1.0018 \mathrm{~g} / \mathrm{L}$ ) was added. Regarding the volatile composition, the stock solution was composed of 307,000 $\mu \mathrm{g} / \mathrm{L}$ of ethyl acetate, $1140 \mu \mathrm{g} / \mathrm{L}$ of isobutyl acetate, $5540 \mu \mathrm{g} / \mathrm{L}$ of ethyl butanoate, $164,000 \mu \mathrm{g} / \mathrm{L}$ of 2-methyl-1-propanol, 2,022,000 $\mu \mathrm{g} / \mathrm{L}$ of isopentyl acetate, $9590 \mu \mathrm{g} / \mathrm{L}$ of 1-butanol, 5,648,000 $\mathrm{gg} / \mathrm{L}$ of isopentyl alcohol, 832,000 $\mu \mathrm{g} / \mathrm{L}$ of ethyl hexanoate, $4088 \mu \mathrm{g} / \mathrm{L}$ of styrene, $4830 \mu \mathrm{g} / \mathrm{L}$ of hexyl acetate, $1018 \mu \mathrm{g} / \mathrm{L}$ of ethyl 3-hexenoate, $312 \mu \mathrm{g} / \mathrm{L}$ of 2-heptanol, $464 \mu \mathrm{g} / \mathrm{L}$ of ethyl enanthate, $368 \mu \mathrm{g} / \mathrm{L}$ of 6-methyl-5-heptene-2-one, $204 \mathrm{mg} / \mathrm{L}$ of ethyl lactate, 2,021,000 $\mu \mathrm{g} / \mathrm{L}$ of 1-hexanol, $8350 \mu \mathrm{g} / \mathrm{L}$ of 3-hexen-1-ol, $1560 \mu \mathrm{g} / \mathrm{L}$ of caprylic acid methyl ester, $910 \mu \mathrm{g} / \mathrm{L}$ of nonanal, 816,000 $\mu \mathrm{g} / \mathrm{L}$ of ethyl caprylate, $1650 \mu \mathrm{g} / \mathrm{L}$ of 1-octen-3-ol, $1049 \mu \mathrm{g} / \mathrm{L}$ of acetic acid, $352 \mu \mathrm{g} / \mathrm{L}$ of sulcatol, $5000 \mu \mathrm{g} / \mathrm{L}$ of isopentyl hexanoate, $5376 \mu \mathrm{g} / \mathrm{L}$ of furfural, $900 \mu \mathrm{g} / \mathrm{L}$ of 2-ethyl-1-hexanol, $2530 \mu \mathrm{g} / \mathrm{L}$ of decanal, $770 \mu \mathrm{g} / \mathrm{L}$ of ethyl nonanoate, $2270 \mu \mathrm{g} / \mathrm{L}$ of ethyl 2-hydroxy-4-methylpentanoate, $1650 \mu \mathrm{g} / \mathrm{L}$ of 1-octanol, $1273 \mathrm{mg} / \mathrm{L}$ of 2,3-Butanediol, $\left[S-\left(R^{*}, R^{*}\right)\right]$-, $668 \mu \mathrm{g} / \mathrm{L}$ of trans-2-octenol, $2030 \mu \mathrm{g} / \mathrm{L}$ of butanoic acid, $81.6 \mu \mathrm{g} / \mathrm{L}$ of ethyl caprate, $324 \mu \mathrm{g} / \mathrm{L}$ of isoamyl caprylate, $79.2 \mu \mathrm{g} / \mathrm{L}$ of diethyl succinate, $2240 \mu \mathrm{g} / \mathrm{L}$ of methionol, $484 \mu \mathrm{g} / \mathrm{L}$ of naphthalene, $4352 \mu \mathrm{g} / \mathrm{L}$ of ethyl salicylate, $1140 \mu \mathrm{g} / \mathrm{L}$ of phenethyl acetate, $10.66 \mu \mathrm{g} / \mathrm{L}$ of ethyl laurate, $119 \mu \mathrm{g} / \mathrm{L}$ of dihydropseudoionone, $1.172 \mu \mathrm{g} / \mathrm{L}$ of bnenzyl alcohol, $81.2 \mu \mathrm{g} / \mathrm{L}$ of phenylethyl alcohol, $18.92 \mu \mathrm{g} / \mathrm{L}$ of octanoic acid, $1600 \mu \mathrm{g} / \mathrm{L}$ of 4 -ethylphenol, and $18.36 \mu \mathrm{g} / \mathrm{L}$ of decanoic acid. The resultant solution was diluted into fourteen successive levels and extracted using the same headspace solid-phase micro-extraction method in the wine sample to generate standard curve (peak area ratio of volatile 
standard over internal standard versus concentration of volatile standard). The quantitation of the volatile compound was based on their corresponding standard. For the volatile without the available standard, the quantitation was conducted using the volatile standard that possessed the similar chemical structure or similar C atom numbers (Table 2).

\subsection{Odor Activity Value and Aroma Series}

Odor activity value (OAV) is an indicator to evaluate the contribution of volatile compound to the overall aroma of wine. The OAV value was calculated as the ratio of the concentration of an individual volatile in wine to its perception threshold.

The volatile compounds were divided into six aromatic groups regarding their odor description $[28,37,38,46]$. These volatile compounds were described to possess: (1) fatty, (2) caramel, (3) fruity, (4) herbaceous (or vegetal), (5) floral, and (6) chemical odors. The total aroma intensity of each aroma group was calculated by summing up the OAVs of each volatile in the group [28].

\subsection{Statistical Analysis}

The difference of mean caused by different goji raw materials (dried goji berry versus fresh goji berry) was assessed using T-test, whereas two-way analysis of variance was performed to investigate the effect of pressing and glycosidase on the volatile concentration difference using SPSS 21.0 (Chicago, IL, USA). Principal component analysis (PCA) was carried out on MetaboAnalyst 3.0 (http:/ / www. metaboanalyst.ca/) using the volatile compounds with the concentration being statistically different among the samples as the variables [52]. Before the PCA analysis, these variables were normalized by autoscaling.

\section{Conclusions}

In conclusion, goji berry wine was produced using as raw material fresh and dried goji berry fruits and the effect of pressing and glycosidase addition with regard to the aromatic composition in the wine was investigated. Dried goji berry fermented wine contained more odor active volatiles and expressed stronger aromatic features including floral, fruity, and caramel odors. The main aromatic character in goji berry fermented wine included floral, fruity, and caramel odors, whereas a stronger chemical note was found in fresh goji berry fermented wine. Goji berry wine made of free-run juice exhibited a better aromatic features compared to wine fermented from the pressed juice. Glycosidase did not show any beneficial effects on the aromatic profile of goji berry fermented wine.

Acknowledgments: This study was financially supported by the Fundamental Research Funds for the Central Universities (2015ZCQ-SW-04 and NO. YX2015-15). All the authors thank Ningxia Senmiao Goji Technology Development Co. Ltd. for their support for goji berry wine making.

Author Contributions: B.-Q.Z. and B.-L.Z. designed the research; G.Y., J.R., L.W., M.W., X.S. and X.O. performed the research; G.Y. and B.-Q.Z. analyzed the data; G.Y. and B.-Q.Z. wrote the paper. All authors read and approved the final manuscript.

Conflicts of Interest: The authors declare no conflict of interest. The founding sponsors had no role in the design of the study; in the collection, analyses, or interpretation of data; in the writing of the manuscript, and in the decision to publish the results.

\section{References}

1. Dong, J.Z.; Wang, Y.; Wang, S.H.; Yin, L.P.; Xu, G.J.; Zheng, C.; Lei, C.; Zhang, M.Z. Selenium increases chlorogenic acid, chlorophyll and carotenoids of lycium chinense leaves. J. Sci. Food. Agric. 2013, 93, 310-315. [CrossRef] [PubMed]

2. Inbaraj, B.S.; Lu, H.; Hung, C.F.; Wu, W.B.; Lin, C.L.; Chen, B.H. Determination of carotenoids and their esters in fruits of lycium barbarum linnaeus by hplc-dad-apci-ms. J. Pharmaceut. Biomed. 2008, 47, 812-818. [CrossRef] [PubMed] 
3. Amagase, H.; Nance, D.M. Lycium barbarum increases caloric expenditure and decreases waist circumference in healthy overweight men and women: Pilot study. J. Am Coll. Nurt. 2011, 30, 304-309. [CrossRef]

4. Donno, D.; Mellano, M.; Raimondo, E.; Cerutti, A.; Prgomet, Z.; Beccaro, G. Influence of applied drying methods on phytochemical composition in fresh and dried goji fruits by hplc fingerprint. Eur Food Res. Technol. 2016, 1-14. [CrossRef]

5. $\quad$ Tang, W.-M.; Chan, E.; Kwok, C.-Y.; Lee, Y.-K.; Wu, J.-H.; Wan, C.-W.; Chan, R.Y.-K.; Yu, P.H.-F.; Chan, S.-W. A review of the anticancer and immunomodulatory effects of Lycium barbarum fruit. Inflammopharmacology 2012, 20, 307-314. [CrossRef] [PubMed]

6. Zhong, Y.; Shahidi, F.; Naczk, M. Phytochemicals and health benefits of goji berries. In Dried Fruits: Phytochemicals and Health Effects; Alasalvar, C., Shahidi, F., Eds.; Blackwell Publishing Ltd.: Oxford, UK, 2013; pp. 133-144.

7. Antalick, G.; Šuklje, K.; Blackman, J.W.; Meeks, C.; Deloire, A.; Schmidtke, L.M. Influence of grape composition on red wine ester profile: Comparison between cabernet sauvignon and shiraz cultivars from australian warm climate. J. Agric. Food Chem. 2015, 63, 4664-4672. [CrossRef] [PubMed]

8. Kennedy, J.F.; Kaczmarek, A. Handbook of enology, the chemistry of wine stabilization and treatments, 2nd ed.; Ribéreau-gayon, P., Glories, Y., Maujean, A., Dubourdieu, D., Eds.; John wiley \& sons, Ltd.: New York, NY, USA, 2006; Volume 2.

9. Cabaroglu, T.; Selli, S.; Canbas, A.; Lepoutre, J.-P.; Günata, Z. Wine flavor enhancement through the use of exogenous fungal glycosidases. Enzyme. Microb Tech. 2003, 33, 581-587. [CrossRef]

10. Selli, S.; Bagatar, B.; Sen, K.; Kelebek, H. Evaluation of differences in the aroma composition of free-run and pressed neutral grape juices obtained from emir (vitis vinifera L.). Chem. Biodivers. 2011, 8, 1776-1782. [CrossRef] [PubMed]

11. Fundira, M.; Blom, M.; Pretorius, I.; Rensburg, P. Comparison of commercial enzymes for the processing of marula pulp, wine, and spirits. J. Food Sci. 2002, 67, 2346-2351. [CrossRef]

12. Palomo, E.S.; Hidalgo, M.C.D.-M.; Gonzalez-Vinas, M.; Pérez-Coello, M. Aroma enhancement in wines from different grape varieties using exogenous glycosidases. Food Chem. 2005, 92, 627-635. [CrossRef]

13. Li, X.; Lim, S.L.; Yu, B.; Curran, P.; Liu, S.Q. Impact of pulp on the chemical profile of mango wine. S. Afr. J. Enol. Vitic. 2013, 34, 119-128.

14. Patel, P.; Herbst-Johnstone, M.; Lee, S.A.; Gardner, R.C.; Weaver, R.; Nicolau, L.; Kilmartin, P.A. Influence of juice pressing conditions on polyphenols, antioxidants, and varietal aroma of sauvignon blanc microferments. J. Agric. Food Chem. 2010, 58, 7280-7288. [CrossRef] [PubMed]

15. Amagase, H.; Farnsworth, N.R. A review of botanical characteristics, phytochemistry, clinical relevance in efficacy and safety of Lycium barbarum fruit (goji). Food Res. Int. 2011, 44, 1702-1717. [CrossRef]

16. Guadalupe, Z.; Ayestarán, B.; Williams, P.; Doco, T. Determination of must and wine polysaccharides by gas chromatography-mass spectrometry (gc-ms) and size-exclusion chromatography (sec). In Polysaccharides: Bioactivity and Biotechnology; Ramawat, G.K., Mérillon, J.-M., Eds.; Springer International Publishing: Cham, Switzerland, 2015; pp. 1265-1297.

17. Cliff, M.A.; Pickering, G.J. Determination of odour detection thresholds for acetic acid and ethyl acetate in ice wine. J. Wine Res. 2006, 17, 45-52. [CrossRef]

18. Panceri, C.P.; De Gois, J.S.; Borges, D.L.G.; Bordignon-Luiz, M.T. Effect of grape dehydration under controlled conditions on chemical composition and sensory characteristics of cabernet sauvignon and merlot wines. LWT-Food Sci. Technol. 2015, 63, 228-235. [CrossRef]

19. Ni, Z.-J.; He, L.; Min, C. Changes of the main carotenoid pigment contents during the drying processes of the different harvest stage fruits of Lycium barbarum L. Agric. Sci. China 2008, 7, 363-369.

20. Wang, D.; Cai, J.; Zhu, B.-Q.; Wu, G.-F.; Duan, C.-Q.; Chen, G.; Shi, Y. Study of free and glycosidically bound volatile compounds in air-dried raisins from three seedless grape varieties using hs-spme with gc-ms. Food Chem. 2015, 177, 346-353. [CrossRef] [PubMed]

21. Lan, Y.-B.; Qian, X.; Yang, Z.-J.; Xiang, X.-F.; Yang, W.-X.; Liu, T.; Zhu, B.-Q.; Pan, Q.-H.; Duan, C.-Q. Striking changes in volatile profiles at sub-zero temperatures during over-ripening of 'beibinghong' grapes in northeastern china. Food Chem. 2016, 212, 172-182. [CrossRef] [PubMed]

22. Guiné, R.P.; Martín-Belloso, O.; Mínguez-Mosquera, M.I.; Paliyath, G.; Pessoa, F.L.; Le Quéré, J.-L.; Sidhu, J.S.; Sinha, N.; Peggy Stanfield, R.; Hui, Y. Handbook of Fruit and Vegetable Flavors; John Wiley and Sons: New York, NY, USA, 2010. 
23. de Lerma, N.L.; Moreno, J.; Peinado, R.A. Determination of the optimum sun-drying time for Vitis vinifera L. cv. Tempranillo grapes by e-nose analysis and characterization of their volatile composition. Food. Bioprocess Tech. 2014, 7, 732-740. [CrossRef]

24. Romano, P. Metabolic characteristics of wine strains during spontaneous and inoculated fermentation. Food Technol. Biotech. 1997, 35, 255-260.

25. Ruiz, M.J.; Zea, L.; Moyano, L.; Medina, M. Aroma active compounds during the drying of grapes cv. Pedro ximenez destined to the production of sweet sherry wine. Eur. Food Res. Technol. 2010, 230, 429-435. [CrossRef]

26. Chung, T.-Y.; Hayase, F.; Kato, H. Volatile components of ripe tomatoes and their juices, purees and pastes. Agric. Biol. Chem. 1983, 47, 343-351.

27. Ho, C.-T.; Chen, Q. Lipids in food flavors: An overview. In Proceedings of 205th National Meeting of the American Chemical Society, Denver, Colorado, 28 March-2 April 1993; American Chemical Society: Washington, DC, USA, 1994.

28. Cai, J.; Zhu, B.-Q.; Wang, Y.-H.; Lu, L.; Lan, Y.-B.; Reeves, M.J.; Duan, C.-Q. Influence of pre-fermentation cold maceration treatment on aroma compounds of cabernet sauvignon wines fermented in different industrial scale fermenters. Food Chem. 2014, 154, 217-229. [CrossRef] [PubMed]

29. Swiegers, J.H.; Pretorius, I.S. Yeast modulation of wine flavor. Adv. Appl. Microbiol. 2005, 57, 131-175. [PubMed]

30. Sánchez-Palomo, E.; Gómez García-Carpintero, E.; Alonso-Villegas, R.; González-Viñas, M. Characterization of aroma compounds of verdejo white wines from the la mancha region by odour activity values. Flavour Frag. J. 2010, 25, 456-462. [CrossRef]

31. Noguerol-Pato, R.; González-Álvarez, M.; González-Barreiro, C.; Cancho-Grande, B.; Simal-Gándara, J. Aroma profile of garnacha tintorera-based sweet wines by chromatographic and sensorial analyses. Food Chem. 2012, 134, 2313-2325. [CrossRef] [PubMed]

32. Noguerol-Pato, R.; González-Álvarez, M.; González-Barreiro, C.; Cancho-Grande, B.; Simal-Gándara, J. Evolution of the aromatic profile in garnacha tintorera grapes during raisining and comparison with that of the naturally sweet wine obtained. Food Chem. 2013, 139, 1052-1061. [CrossRef] [PubMed]

33. Sumby, K.M.; Grbin, P.R.; Jiranek, V. Microbial modulation of aromatic esters in wine: Current knowledge and future prospects. Food Chem. 2010, 121, 1-16. [CrossRef]

34. Coghe, S.; Benoot, K.; Delvaux, F.; Vanderhaegen, B.; Delvaux, F.R. Ferulic acid release and 4-vinylguaiacol formation during brewing and fermentation: Indications for feruloyl esterase activity in saccharomyces cerevisiae. J. Agric. Food Chem. 2004, 52, 602-608. [CrossRef] [PubMed]

35. Dias, L.; Dias, S.; Sancho, T.; Stender, H.; Querol, A.; Malfeito-Ferreira, M.; Loureiro, V. Identification of yeasts isolated from wine-related environments and capable of producing 4-ethylphenol. Food Microbiol. 2003, 20, 567-574. [CrossRef]

36. Kanasawud, P.; Crouzet, J.C. Mechanism of formation of volatile compounds by thermal degradation of carotenoids in aqueous medium. 1.. Beta.-carotene degradation. J. Agric. Food Chem. 1990, 38, 237-243. [CrossRef]

37. Wu, Y.; Zhu, B.; Tu, C.; Duan, C.; Pan, Q. Generation of volatile compounds in litchi wine during winemaking and short-term bottle storage. J. Agric. Food Chem. 2011, 59, 4923-4931. [CrossRef] [PubMed]

38. Zhu, W.; Zhu, B.; Li, Y.; Zhang, Y.; Zhang, B.; Fan, J. Acidic electrolyzed water efficiently improves the flavour of persimmon (diospyros kaki l. Cv. Mopan) wine. Food Chem. 2016, 197(Part A), 141-149. [CrossRef] [PubMed]

39. Apostolopoulou, A.; Flouros, A.; Demertzis, P.; Akrida-Demertzi, K. Differences in concentration of principal volatile constituents in traditional greek distillates. Food Control. 2005, 16, 157-164. [CrossRef]

40. Satora, P.; Tuszyński, T. Influence of indigenous yeasts on the fermentation and volatile profile of plum brandies. Food Microbiol. 2010, 27, 418-424. [CrossRef] [PubMed]

41. Baek, H.; Cadwallader, K. Contribution of free and glycosidically bound volatile compounds to the aroma of muscadine grape juice. J. Food Sci. 1999, 64, 441-444. [CrossRef]

42. Chatonnet, P.; Dubourdie, D.; Boidron, J.; Pons, M. The origin of ethylphenols in wines. J. Sci. Food. Agric. 1992, 60, 165-178. [CrossRef]

43. Towey, J.P.; Waterhouse, A.L. The extraction of volatile compounds from french and american oak barrels in chardonnay during three successive vintages. Am. J. Enol. Viticult. 1996, 47, 163-172. 
44. Pollnitz, A.P.; Pardon, K.H.; Sefton, M.A. Quantitative analysis of 4-ethylphenol and 4-ethylguaiacol in red wine. J. Chromatogr. A 2000, 874, 101-109. [CrossRef]

45. Sun, S.Y.; Gong, H.S.; Jiang, X.M.; Zhao, Y.P. Selected non-saccharomyces wine yeasts in controlled multistarter fermentations with saccharomyces cerevisiae on alcoholic fermentation behaviour and wine aroma of cherry wines. Food Microbiol. 2014, 44, 15-23. [CrossRef] [PubMed]

46. Noble, A.C.; Arnold, R.A.; Buechsenstein, J.; Leach, E.J.; Schmidt, J.; Stern, P.M. Modification of a standardized system of wine aroma terminology. Am. J. Enol. Viticult. 1987, 38, 143-146.

47. Ferreira, V.; López, R.; Cacho, J.F. Quantitative determination of the odorants of young red wines from different grape varieties. J. Sci. Food. Agric. 2000, 80, 1659-1667. [CrossRef]

48. Pino, J.A.; Queris, O. Analysis of volatile compounds of mango wine. Food Chem. 2011, 125, 1141-1146. [CrossRef]

49. Guth, H. Quantitation and sensory studies of character impact odorants of different white wine varieties. J. Agric. Food Chem. 1997, 45, 3027-3032. [CrossRef]

50. Zhao, N.; Wen, Y.; Pan, Q. Determination of dry red wine polysaccharide by phenol-sulfuric acid method. Sino-Overseas Grapevine Wine 2011, 5, 003. [CrossRef]

51. Zhang, M.; Xu, Q.; Duan, C.; Qu, W.; Wu, Y. Comparative study of aromatic compounds in young red wines from cabernet sauvignon, cabernet franc, and cabernet gernischet varieties in china. J. Food Sci. 2007, 72, 248-252. [CrossRef] [PubMed]

52. Xia, J.; Sinelnikov, I.V.; Han, B.; Wishart, D.S. Metaboanalyst 3.0-Making metabolomics more meaningful. Nucleic Acids Res. 2015, 43, 251-257. [CrossRef] [PubMed]

Sample Availability: Some of the frozen wine samples are available from the authors.

(C) 2016 by the authors; licensee MDPI, Basel, Switzerland. This article is an open access article distributed under the terms and conditions of the Creative Commons Attribution (CC-BY) license (http://creativecommons.org/licenses/by/4.0/). 